\title{
A Generalised Formulation for Computing the Microbuckling Load in Periodic Layered Materials
}

\author{
I.A. Guz, M.V. Menshykova, O.V. Menshykov \\ Centre for Micro- and Nanomechanics (CEMINACS) \\ School of Engineering, University of Aberdeen \\ Aberdeen AB24 3UE, Scotland
}

\begin{abstract}
The paper develops a generalised approach to instability of layered materials for numerical realisation of the method as applied to various constitutive equations of the layers (elastic, hyperelastic, elastic-plastic), different loading schemes (uniaxial or biaxial loading) and different precritical conditions (large or small precritical deformations). It contains many examples of calculations of critical controlled parameters for particular model materials as well as the analysis of different buckling modes.
\end{abstract}

Keywords: Layered materials; Microstructure; Microbuckling; Analytical solution; Compression 


\section{Introduction}

\subsection{Background}

The compressive strength is often a design-limiting factor for advanced layered materials: it is generally $30-40 \%$ lower than the tensile strength [1-4]. A better understanding of the compressive strength and failure mechanisms is therefore fundamental to the development of improved materials.

The work [5] was the first to consider the microbuckling of fibres as a form of fracture of a unidirectionally reinforced material undergoing compression. Since then, the beginning of fracture process under compression is usually associated with the buckling of the microstructure of the material when the critical load is determined by parameters characterising the microstructure rather than by the dimensions and shape of the specimen or structural member, i.e. with the internal instability phenomena according to [6]. In this paper we adopt the same assumption of linking the onset of fracture and the loss of stability in the internal structure of the material. The task of deriving three-dimensional (3-D) analytical solutions to describe the response of layered materials was always considered as one of great importance [7]. Analytical solutions, if obtained, enable us to analyse the behaviour of a structure over the wide range of material properties, and loading schemes, without the restrictions imposed by simplified approximate methods.

This paper re-visits the exact 3-D approach to study internal instability in layered media, when the behaviour of each component of the material is described by 3-D equations of solid mechanics.

\subsection{A brief review of analytical studies of internal instability for layered materials}

Probably the first solutions to the problem of internal instability for a layered material obtained within this approach were reported in [8-10], where the problem for linear-elastic layers under uniaxial compression was solved. This solution was included in numerous books and the comprehensive review on the topic [11]. Later the exact solutions were derived also for more complex problems: for orthotropic, non-linear elastic and elastic-plastic, compressible and incompressible layers including the case of large (finite) deformations - see, for example, [15-18] and the reviews $[11,19]$.

The importance and the complexity of the considered phenomena caused a large number of publications which put forward various approximate methods aimed at tackling the problems with different levels of accuracy: the early papers [20-22] and the numerous later publications reviewed 
in [1-4]. It was concluded after the detailed analyses [2, 4, 11], that the approximate methods are not very accurate when compared to experimental measurements and observations.

For instance, one of the earlier models suggested in [20] involves considerable simplifications, modelling the reinforcement layers by the thin beam theory and the matrix as an elastic material using one-dimensional stress analysis. It makes the results of this method inaccurate even for simple cases. It was shown [11, 23, 24] that the approximate model can give a significant discrepancy in comparison with the exact approach and with experimental data. Another approach, which is commonly used, is based on the investigation of fibre kinking. From the early literature on compressive fracture it was easy to get the impression that fibre instability (microbuckling) and kinking are competing mechanisms. However, it is now accepted that a kink band is an outcome of the microbuckling failure of actual fibres, as observed experimentally [25, 26]. Fibre microbuckling occurs first, followed by propagation of this local damage to form a kink band. A comprehensive comparative analysis of the Rosen model, Argon-Budiansky (kinking) model, and Batdorf-Ko model was presented in [2]. Studies of the kinking phenomenon were also reviewed in [1]. It was shown [2] that the existing kinking analyses are able to account for some, but not all, of the experimental observations. They correctly predict that shear strength and fibre imperfections are important parameters affecting the compressive strength of composite materials. However, within this model it is not possible to say exactly how the strength will vary with fibre content; and the value of misalignment is chosen arbitrarily. This model requires knowledge of the shear strength properties, the initial fibre misalignment and, the most importantly, the kink-band orientation angle which is a post-failure geometric parameter. The analysis of this approach is outside the scope of the present paper, but it is worth noting that the works mentioned above considered perfectly bonded layers only. Moreover, the approaches based on the model [20] and the kink-band model cannot be altogether applied in the cases of large pre-critical deformations (such as those considered in Section 4.2 of the present paper).

This paper is concerned with the development of a unified procedure for realisation of the 3-D analytical method as applied to various constitutive equations of the layers (incompressible hyperelastic, compressible linear elastic, incompressible elastic-plastic), different loading schemes (uniaxial or biaxial compression) and different precritical conditions (large or small precritical deformations). It contains many examples of calculation of critical strains and shortening factors for generic layered materials as well as the analysis of different buckling modes. The use of 3-D stability theory places the method into the category of "exact" approaches, as opposed to approximate models based on certain simplifications when describing the stress-strain state. Some comparisons of the results obtained within this approach with the available experimental data were 
discussed earlier in [11, 27, 28], however the purpose of this work is to present a procedure which uniformly deals with different types of layered materials. Therefore we have intentionally chosen to present the computations for model generic material systems only. The results of this approach can be used as a benchmark for simplified analytical models.

\section{The problem formalism within the 3-D theory of stability}

Let us briefly consider the statement of the problem of internal instability (microbuckling) for layered materials. The detailed formulations for the particular types of layers were given, for example, in [17, 18, 23].

The material consists of alternating layers with thicknesses $2 h_{r}$ and $2 h_{m}$. Two different loading schemes are studied: the uniaxial compression and the biaxial compression in the plane of the layers, Fig. 1. The solution of the problem is sought for four modes of stability loss (Fig. 2), see for example, [17]. Using the equations of the 3-D stability theory [29] the following eigen-value problem must be solved:

The stability equations for each layer are [29]:

$$
\frac{\partial}{\partial x_{i}} t_{i j}^{r}=0, \quad \frac{\partial}{\partial x_{i}} t_{i j}^{m}=0 ; \quad i, j=1,2,3,
$$

where $t_{i j}$ is the non-symmetrical Piola-Kirchhoff stress tensor (nominal stress tensor).

Tensor $t_{i j}$ has the following form for incompressible solids [29]:

$$
t_{i j}=\kappa_{i j \alpha \beta} \frac{\partial u_{\alpha}}{\partial x_{\beta}}+\delta_{i j} \lambda_{j}^{-1} p,
$$

where

$$
\lambda_{1} \lambda_{2} \lambda_{3}=1
$$

is the incompressibility condition, $\lambda_{j}$ is the elongation/shortening factor in the direction of the $O X_{j}$ axis).

For compressible solids [29]:

$$
t_{i j}=\omega_{i j \alpha \beta} \frac{\partial u_{\alpha}}{\partial x_{\beta}} .
$$

The components of the tensors $\kappa_{i j \alpha \beta}$ and $\omega_{i j \alpha \beta}$ depend on the properties of the layers and the loads. The most general expressions for $\kappa_{i j \alpha \beta}$ and $\omega_{i j \alpha \beta}$ could be found in [29]: 


$$
\begin{gathered}
\kappa_{i j \alpha \beta}=\lambda_{j} \lambda_{\alpha}\left[\delta_{i j} \delta_{\alpha \beta} A_{\beta i}+\left(1-\delta_{i j}\right)\left(\delta_{i \alpha} \delta_{j \beta} \mu_{i j}+\delta_{i \beta} \delta_{j \alpha} \mu_{j i}\right)\right]+\delta_{i \beta} \delta_{j \alpha} S_{\beta \beta}^{0}, \\
\omega_{i j \alpha \beta}=\lambda_{j} \lambda_{\alpha}\left[\delta_{i j} \delta_{\alpha \beta} A_{\beta i}^{\prime}+\left(1-\delta_{i j}\right)\left(\delta_{i \alpha} \delta_{j \beta} \mu_{i j}^{\prime}+\delta_{i \beta} \delta_{j \alpha} \mu_{j i}^{\prime}\right)\right]+\delta_{i \beta} \delta_{j \alpha} S_{\beta \beta}^{0},
\end{gathered}
$$

where $A_{i j}\left(A_{i j}^{\prime}\right)$ and $\mu_{i j}\left(\mu_{i j}^{\prime}\right)$ are the quantities which characterise the axial and shear stiffnesses. The quantity characterising the precritical state (the stress component $S_{11}^{0}$ or the strain component $\varepsilon_{11}^{0}$ ) is the parameter in respect to which the eigen-value problem should be solved.

To complete the problem statement, the boundary conditions should be defined for each interface. The layer interfaces could consist of zones of perfectly connected (bonded) layers and defects such as cracks or delaminations. In this study we consider materials with either perfectly bonded layers or "perfectly lubricated" (sliding without friction) interfaces when a tangential slip is allowed at the interface between the layers. In the latter case, a change in the nature of the interlaminar contact occurs, when an interaction of the layers is implemented so, that infinitesimal sliding is allowed, but still there are no gaps between the layers, e.g., molecular chains in some kinds of glue connection. This special kind of a slip-type delamination is sometimes called "defects with connected edges”, or, according to [30, 31], “perfectly lubricated interfaces”. For the perfectly bonded layers we have the continuity conditions for the stresses and displacements

$$
t_{3 i}^{r}=t_{3 i}^{m}, u_{i}^{r}=u_{i}^{m}, \quad i=\overline{1,3} .
$$

For the perfectly lubricated interfaces [31] only the transversal displacement and the normal traction remain continuous although the shear tractions at the respective interface vanish: complete slip without friction in the tangential direction is allowed. The boundary conditions for perturbations of stresses and displacements become

$$
t_{31}^{r}=t_{31}^{m}=t_{32}^{r}=t_{32}^{m}=0, t_{33}^{r}=t_{33}^{m}, u_{3}^{r}=u_{3}^{m} .
$$

Note that in practical cases the assumption of perfect bonding between neighbouring layers does not correspond to reality due to different imperfections always present in real layered materials. When considering a material with such defects it is sometimes difficult to identify a set of the defects and its influence on the onset of instability. Hence, we suggest the following estimation. It is obvious that the critical strain $\varepsilon_{c r}$ for a material with imperfections of interfacial adhesion must be larger than the critical strain $\varepsilon_{c r}^{p l}$ for the same material with perfectly lubricated layers, but smaller than the critical strain $\varepsilon_{c r}^{p b}$ for the structure with perfectly bonded layers. Thus, we obtain the following bounds for the critical strain:

$$
\varepsilon_{c r}^{p l} \leq \varepsilon_{c r} \leq \varepsilon_{c r}^{p b}
$$




\section{Deriving analytical solutions}

Solutions of equation (1) (i.e. perturbations of stresses and displacements) for each of the layers can be expressed through the functions $\mathrm{X}$ and $\Psi$, which are the solutions of the following equations [29]

$$
\begin{gathered}
\left(\Delta_{1}+\xi_{1}^{2} \frac{\partial^{2}}{\partial x_{3}^{2}}\right) \Psi=0 \\
\left(\Delta_{1}+\xi_{2}^{2} \frac{\partial^{2}}{\partial x_{3}^{2}}\right)\left(\Delta_{1}+\xi_{3}^{2} \frac{\partial^{2}}{\partial x_{3}^{2}}\right) X=0,
\end{gathered}
$$

where

$$
\Delta_{1}=\frac{\partial^{2}}{\partial x_{1}^{2}}+\frac{\partial^{2}}{\partial x_{2}^{2}} .
$$

The parameter $\xi_{j}$ depends on the components of the tensor $\kappa_{i j \alpha \beta}$ (or $\omega_{i j \alpha \beta}$ ) and, therefore, on the properties of the layers and on the loads. It was proved in [12, 13] that for elastic compressible and elastic incompressible layers

$$
\xi_{j}^{2}>0, \operatorname{Im} \xi_{j}^{2}=0
$$

and for elastic-plastic incompressible layers

$$
\operatorname{Im} \xi_{2,3}^{2} \neq 0, \xi_{3}^{2}={\overline{\xi_{2}^{2}}}^{2}
$$

The characteristic determinants associated with the four modes of stability loss (Fig. 2) were derived earlier in [11-14] for various constitutive equations of the layers, different loading schemes (uniaxial or biaxial loading) and different precritical conditions (large or small precritical deformations). The considered modes of stability loss include all possible periodic modes with periods, which are equal to one or two periods of the internal structure. Similarly, characteristic equations can be derived for other modes of stability loss. The described method can give the solutions for modes with periods, which are equal to $3,4,5, \ldots$ periods of the internal structure. Other modes with periods, which are not multiples of the period of the internal structure, can also be examined. The solution for them would be based either on the Floquet theorem for ordinary differential equations with periodic coefficients, or on reducing the problem to an infinite set of equations with the consequent solution by a numerical method [11]. However, the modes with the larger periods in transverse direction are usually not of practical interest $[11,17,18]$. In this paper, 
the characteristic determinants are presented in the unified form in order to facilitate a uniform computational procedure for solving them:

- for perfectly bonded layers

$$
\operatorname{det}\left\|\begin{array}{llll}
\beta_{11} & \beta_{12} & \beta_{13} & \beta_{14} \\
\beta_{21} & \beta_{22} & \beta_{23} & \beta_{24} \\
\beta_{31} & \beta_{32} & \beta_{33} & \beta_{34} \\
\beta_{41} & \beta_{42} & \beta_{43} & \beta_{44}
\end{array}\right\|=0,
$$

- for perfectly lubricated layers

$$
\operatorname{det}\left\|\begin{array}{cccc}
\beta_{11} & \beta_{12} & 0 & 0 \\
\beta_{21} & \beta_{22} & \beta_{23} & \beta_{24} \\
0 & 0 & \beta_{13} & \beta_{14} \\
\beta_{41} & \beta_{42} & \beta_{43} & \beta_{44}
\end{array}\right\|=0 .
$$

The expressions for $\beta_{i j}$ of the determinant for different types of materials and for different loading schemes are given in [11, 15-17, 23, 24, 32].

\section{A unified computational procedure for different periodic layered materials}

\subsection{Procedure for computing the critical controlled parameters}

To facilitate the analysis of characteristic determinants, the software package with the graphical user-friendly interface was developed using MATLAB 7.6.0. The software contains a database of properties for typical layered materials and a library of components of tensors $\kappa_{i j \alpha \beta}$ and $\omega_{i j \alpha \beta}$, Eqs. (5), (6). The fully automated numerical procedure consists of the following steps. First, the characteristic determinants, Eqs. (15) and (16), are computed depending on the user's choice of loading schemes (uniaxial or biaxial loading), initial conditions (large or small precritical deformations), and interfacial properties (perfectly bonded and perfectly lubricated layers). Then the results are analysed, and the critical controlled parameters of the internal instability (including the critical wavelength) are searched for. This analysis is conducted for all four considered modes of stability loss. At the final stage the modes are compared and the critical mode is found.

Some of the results for the cases of perfectly bonded and perfectly lubricated layers are presented in the following subsections of this paper.

\subsection{Hyperelastic incompressible layered materials}


Here the material under consideration consists of alternating non-linear hyperelastic layers - many new materials fall into this category, see [33]. Suppose that the materials of these layers are incompressible and a simplified version of the Mooney's potential, namely the so-called neoHookean potential, may be chosen to describe them in the following form

$$
\Phi^{r}=2 C_{10}^{r} I_{1}^{r}\left(\varepsilon_{i j}^{0}\right), \quad \Phi^{m}=2 C_{10}^{m} I_{1}^{m}\left(\varepsilon_{i j}^{0}\right),
$$

where $\Phi$ is the strain energy density function (elastic potential), $\mathrm{C}_{10}$ is a material constant, and $I_{1}(\varepsilon)$ is the first algebraic invariant of the Cauchy-Green strain tensor. This potential is also called the Treloar's potential, after the author who obtained it from an analysis of a model for rubber regarded as a macromolecular network structure made of very long and flexible interlinking chains, see Treloar (1975).

Then the characteristic equations (15) and (16) can be specified for particular modes of stability loss following $[12,17]$. The resulting transcendental equations in terms of $\lambda_{1}$ (shortening factor) and $\alpha_{r}$ (normalised wavelength) will be different for each of the modes. In the case of biaxial loading [17]:

for perfectly bonded layers

- for the first (shear) mode, Fig. 2a,

$$
\begin{aligned}
& -\lambda^{-3}\left(1+\lambda_{1}^{6}\right)^{2}\left[1-C_{10}^{r}\left(C_{10}^{m}\right)^{-1}\right]^{2} \tanh \alpha_{r} \lambda_{1}^{-3} \tanh \alpha_{m} \lambda_{1}^{-3}-4 \lambda_{1}^{3}\left[1-C_{10}^{r}\left(C_{10}^{m}\right)^{-1}\right]^{2} \tanh \alpha_{r} \tanh \alpha_{m} \\
& +\left[2-\left(1+\lambda_{1}^{6}\right) C_{10}^{r}\left(C_{10}^{m}\right)^{-1}\right]^{2} \tanh \alpha_{r} \lambda_{1}^{-3} \tanh \alpha_{m}+\left[1+\lambda_{1}^{6}-2 C_{10}^{r}\left(C_{10}^{m}\right)^{-1}\right]^{2} \tanh \alpha_{r} \tanh \alpha_{m} \lambda_{1}^{-3} \\
& +\left(1-\lambda_{1}^{6}\right)^{2} C_{10}^{r}\left(C_{10}^{m}\right)^{-1}\left(\tanh \alpha_{r} \tanh \alpha_{r} \lambda_{1}^{-3}+\tanh \alpha_{m} \tanh \alpha_{m} \lambda_{1}^{-3}\right)=0,
\end{aligned}
$$

- for the second (extension) mode, Fig. 2b,

$$
\begin{aligned}
& -\lambda^{-3}\left(1+\lambda_{1}^{6}\right)^{2}\left[1-C_{10}^{r}\left(C_{10}^{m}\right)^{-1}\right]^{2} \tanh \alpha_{r} \lambda_{1}^{-3} \operatorname{coth} \alpha_{m} \lambda_{1}^{-3}-4 \lambda_{1}^{3}\left[1-C_{10}^{r}\left(C_{10}^{m}\right)^{-1}\right]^{2} \tanh \alpha_{r} \operatorname{coth} \alpha_{m} \\
& +\left[2-\left(1+\lambda_{1}^{6}\right) C_{10}^{r}\left(C_{10}^{m}\right)^{-1}\right]^{2} \tanh \alpha_{r} \lambda_{1}^{-3} \operatorname{coth} \alpha_{m}+\left[1+\lambda_{1}^{6}-2 C_{10}^{r}\left(C_{10}^{m}\right)^{-1}\right]^{2} \tanh \alpha_{r} \operatorname{coth} \alpha_{m} \lambda_{1}^{-3} \\
& +\left(1-\lambda_{1}^{6}\right)^{2} C_{10}^{r}\left(C_{10}^{m}\right)^{-1}\left(\tanh \alpha_{r} \tanh \alpha_{r} \lambda_{1}^{-3}+\operatorname{coth} \alpha_{m} \operatorname{coth} \alpha_{m} \lambda_{1}^{-3}\right)=0,
\end{aligned}
$$

- for the third mode, Fig. 2c,

$$
\begin{aligned}
& -\lambda^{-3}\left(1+\lambda_{1}^{6}\right)^{2}\left[1-C_{10}^{r}\left(C_{10}^{m}\right)^{-1}\right]^{2} \operatorname{coth} \alpha_{r} \lambda_{1}^{-3} \cot -4 \lambda_{1}^{3}\left[1-C_{10}^{r}\left(C_{10}^{m}\right)^{-1}\right]^{2} \operatorname{coth} \alpha_{r} \operatorname{coth} \alpha_{m} h \alpha_{m} \lambda_{1}^{-3} \\
& +\left[2-\left(1+\lambda_{1}^{6}\right) C_{10}^{r}\left(C_{10}^{m}\right)^{-1}\right]^{2} \operatorname{coth} \alpha_{r} \lambda_{1}^{-3} \operatorname{coth} \alpha_{m}+\left[1+\lambda_{1}^{6}-2 C_{10}^{r}\left(C_{10}^{m}\right)^{-1}\right]^{2} \operatorname{coth} \alpha_{r} \operatorname{coth} \alpha_{m} \lambda_{1}^{-3} \\
& +\left(1-\lambda_{1}^{6}\right)^{2} C_{10}^{r}\left(C_{10}^{m}\right)^{-1}\left(\operatorname{coth} \alpha_{r} \operatorname{coth} \alpha_{r} \lambda_{1}^{-3}+\operatorname{coth} \alpha_{m} \operatorname{coth} \alpha_{m} \lambda_{1}^{-3}\right)=0,
\end{aligned}
$$

- for the fourth mode, Fig. 2d,

$$
\begin{aligned}
& -\lambda^{-3}\left(1+\lambda_{1}^{6}\right)^{2}\left[1-C_{10}^{r}\left(C_{10}^{m}\right)^{-1}\right]^{2} \operatorname{coth} \alpha_{r} \lambda_{1}^{-3} \tanh \alpha_{m} \lambda_{1}^{-3}-4 \lambda_{1}^{3}\left[1-C_{10}^{r}\left(C_{10}^{m}\right)^{-1}\right]^{2} \operatorname{coth} \alpha_{r} \tanh \alpha_{m} \\
& +\left[2-\left(1+\lambda_{1}^{6}\right) C_{10}^{r}\left(C_{10}^{m}\right)^{-1}\right]^{2} \operatorname{coth} \alpha_{r} \lambda_{1}^{-3} \tanh \alpha_{m}+\left[1+\lambda_{1}^{6}-2 C_{10}^{r}\left(C_{10}^{m}\right)^{-1}\right]^{2} \operatorname{coth} \alpha_{r} \tanh \alpha_{m} \lambda_{1}^{-3}=0
\end{aligned}
$$

for perfectly lubricated layers

- for the first (shear) mode, Fig. 2a, 


$$
4 \lambda_{1}^{3}\left(\frac{C_{10}^{r}}{C_{10}^{m}} \tanh \alpha_{r}+\tanh \alpha_{m}\right)-\left(1+\lambda_{1}^{6}\right)^{2}\left(\frac{C_{10}^{r}}{C_{10}^{m}} \tanh \frac{\alpha_{r}}{\lambda_{1}^{3}}+\tanh \frac{\alpha_{m}}{\lambda_{1}^{3}}\right)=0,
$$

- for the second (extension) mode, Fig. 2b,

$$
4 \lambda_{1}^{3}\left(\frac{C_{10}^{r}}{C_{10}^{m}} \tanh \alpha_{r}+\operatorname{coth} \alpha_{m}\right)-\left(1+\lambda_{1}^{6}\right)^{2}\left(\frac{C_{10}^{r}}{C_{10}^{m}} \tanh \frac{\alpha_{r}}{\lambda_{1}^{3}}+\operatorname{coth} \frac{\alpha_{m}}{\lambda_{1}^{3}}\right)=0
$$

- for the third mode, Fig. 2c,

$$
4 \lambda_{1}^{3}\left(\frac{C_{10}^{r}}{C_{10}^{m}} \operatorname{coth} \alpha_{r}+\operatorname{coth} \alpha_{m}\right)-\left(1+\lambda_{1}^{6}\right)^{2}\left(\frac{C_{10}^{r}}{C_{10}^{m}} \operatorname{coth} \frac{\alpha_{r}}{\lambda_{1}^{3}}+\operatorname{coth} \frac{\alpha_{m}}{\lambda_{1}^{3}}\right)=0 .
$$

- for the fourth mode, Fig. 2d,

$$
4 \lambda_{1}^{3}\left(\frac{C_{10}^{r}}{C_{10}^{m}} \operatorname{coth} \alpha_{r}+\tanh \alpha_{m}\right)-\left(1+\lambda_{1}^{6}\right)^{2}\left(\frac{C_{10}^{r}}{C_{10}^{m}} \operatorname{coth} \frac{\alpha_{r}}{\lambda_{1}^{3}}+\tanh \frac{\alpha_{m}}{\lambda_{1}^{3}}\right)=0 .
$$

The shortening factor $\lambda_{1}$ is related to the value of strain $\varepsilon_{11}^{0}$ by the following equation

$$
u_{i}^{0}=\left(\lambda_{i}-1\right) x_{i}, \quad \lambda_{i}=\text { const }, \quad \varepsilon_{i j}^{0}=\left(\lambda_{i}-1\right) \delta_{i j},
$$

where $u_{i}^{0}$ is the axial displacement and $\varepsilon_{i j}^{0}$ is the strain (in terms of the elongation/shortening factor $\lambda_{j}$ in the direction of the $O X_{j}$ axis). The values of displacement and strain corresponding to the precritical state are marked by the superscript ' 0 ' to distinguish them from perturbations of the same values ( $u_{i}^{0}$ and $u_{i}, \varepsilon_{i j}^{0}$ and $\varepsilon_{i j}$ respectively).

In order to obtain the characteristic equations for the uniaxial loading, $\lambda_{1}^{-3}, \lambda_{1}^{3}$, and $\lambda_{1}^{6}$ should be replaced respectively with $\lambda_{1}^{-2}, \lambda_{1}^{2}$, and $\lambda_{1}^{4}$ in Eqs. (18-25).

The critical value for the particular mode, $\lambda_{c r}^{(N)}(N$ is the number of the mode), can be found as a maximum of the corresponding curve. The maximum of these values will be the critical shortening factor of the internal instability for the considered layered material

$$
\begin{aligned}
& \lambda_{c r}^{p l}=\max _{N}\left\{\lambda_{c r}^{(N)}\right\}=\max _{N}\left\{\max _{\alpha_{r}} \lambda_{1}^{(N)}\right\}, \\
& \lambda_{c r}^{p b}=\max _{N}\left\{\lambda_{c r}^{(N)}\right\}=\max _{N}\left\{\max _{\alpha_{r}} \lambda_{1}^{(N)}\right\} .
\end{aligned}
$$

Note that maximum shortening factors correspond to minimal strains and, therefore, to minimal loads according to Eq. (26). The curves corresponding to the $3^{\text {rd }}$ and the $4^{\text {th }}$ modes lie beneath the curves corresponding to the $1^{\text {st }}$ and the $2^{\text {nd }}$ modes, see [17]. Therefore, the $1^{\text {st }}$ and $2^{\text {nd }}$ modes appear to be the most common modes of practical interest.

The computed critical values of shortening factors for hyperelastic materials with perfectly bonded layers under biaxial loading are presented in Figs. 3-5. The comparison of the results for the 
first and second modes of stability loss is presented in Fig. 3 and Fig. 4. The shortening factor tends to zero with the decrease of the material constants ratio and the difference between results for the first and the second modes of stability loss becomes smaller (Fig. 3). Fig. 4 shows how the ratio of the layer thicknesses influences the value of shortening factor for first two modes. The shortening factors for the first and the second modes coincide while the reinforcement layer is thin comparing to the matrix layer. In the considered case the difference between the results for the $1^{\text {st }}$ and the $2^{\text {nd }}$ modes becomes noticeable when the ratio of the layer thicknesses reaches a certain value (0.1 for the case of Fig. 4). It increases with the increase of the ratio of the layer thicknesses.

The 3-D plots in Fig. 5 and Fig. 6 illustrate the combined effect of changes in the ratio of layer thicknesses and the ratio of material constants on the critical shortening factors for the first and the second modes of the stability loss.

\subsection{Compressible linear elastic layered materials}

Let us consider a material consisting of alternating linear-elastic isotropic compressible layers with different elastic properties (the Young's moduli $E$ and the Poisson's ratios $v$ ). Then for the reinforcement layer we have

$$
\left(\sigma_{i j}^{0}\right)^{r}=\delta_{i j} \frac{E_{r} v_{r}}{\left(1+v_{r}\right)\left(1-2 v_{r}\right)} \varepsilon_{n n}^{0}+\frac{E_{r}}{1+v_{r}} \varepsilon_{i j}^{0},
$$

and for the matrix

$$
\left(\sigma_{i j}^{0}\right)^{m}=\delta_{i j} \frac{E_{m} \nu_{m}}{\left(1+v_{m}\right)\left(1-2 v_{m}\right)} \varepsilon_{n n}^{0}+\frac{E_{m}}{1+v_{m}} \varepsilon_{i j}^{0} .
$$

The components of tensor $\omega_{i j \alpha \beta}$ for such materials are given in [23, 29] for different types of loading. Following the procedure described in the previous Subsection, i.e. substituting the expressions for $\omega_{i j \alpha \beta}$ into the characteristic equations (15) and (16), the characteristic equation can be specified for the considered material, see [11, 15, 23] for more details.

For all modes we have the transcendental equations in terms of two variables, $\varepsilon_{11}^{0}$ (applied strain) and $\alpha_{r}$ (normalised half-wavelength). Solving the characteristic equations for different modes of stability loss, the dependences $\varepsilon_{11}^{(N)}\left(\alpha_{r}\right)$ are obtained ( $N=1,2,3,4$ is the number of the mode). A minimum of the corresponding dependence is the critical value for the particular mode $\varepsilon_{c r}^{(N)}$. The critical strain of internal instability for the considered layered material is the minimal of these four values ( $\varepsilon_{c r}^{p l}$ in the case of perfectly lubricated layers, and $\varepsilon_{c r}^{p b}$ in the case of perfectly bonded layers): 


$$
\begin{aligned}
& \varepsilon_{c r}^{p l}=\min _{N} \varepsilon_{c r}^{(N)}=\min _{N}\left(\min _{\alpha_{r}} \varepsilon_{11}^{(N)}\right), \\
& \varepsilon_{c r}^{p b}=\min _{N} \varepsilon_{c r}^{(N)}=\min _{N}\left(\min _{\alpha_{r}} \varepsilon_{11}^{(N)}\right) .
\end{aligned}
$$

The 3-D plots in Figs. 7-10 illustrate the combined effect of changes in the ratio of layer thicknesses and the ratio of Young's moduli for the layers on the critical strains for the $1^{\text {st }}$ and the $2^{\text {nd }}$ modes of stability loss.

The results for materials with perfectly bonded layers under uniaxial loading are shown in Figs 7 and 8. The plots clearly illustrate that an increase in the relative stiffness of reinforcing layers leads to a diminishing critical strain value. The critical strains reaches a maximum value at $\mathrm{E}_{\mathrm{r}} / \mathrm{E}_{\mathrm{m}}=1$, which corresponds to the case of a homogeneous material. This maximum value coincides with the one obtained in [6, 29] for homogeneous isotropic compressible linear elastic materials. This fact is yet another verification of the method. The change in the ratio of layer thicknesses has a nonmonotonous effect on the critical strains. However, after the ratio of layer thicknesses becomes smaller than a certain value (usually around 0.1 ), a further reduction of it would not affect the value of critical strain. In this case the problem is reduced to a linear-elastic layer between two linear elastic half-planes, with the critical strain coinciding with the one obtained analytically for the latter case in [29]. A very high value of critical strain for certain combinations of the material constants and the layer thicknesses is consistent with the results for homogeneous materials [6, 29]. It would never be achieved in a real life situation and such a material would not experience microbuckling in practice.

The results for materials with perfectly lubricated layers under biaxial loading are shown in Figs. 9 and 10. For the $1^{\text {st }}$ mode of stability loss, a decreasing ratio of layer thicknesses leads to a significant decrease in the value of critical strain. For the $2^{\text {nd }}$ mode of stability loss the effect of the ratio of layer thicknesses is non-monotonous. An increase in the relative stiffness of reinforcing layers leads to a smaller critical strain value. This effect is more pronounced for the $2^{\text {nd }}$ mode of stability loss, whereas for the $1^{\text {st }}$ mode the change is rather small.

\subsection{Comparison with an approximate model}

As it was mentioned in Subsection 1.2, one of the earlier models suggested in [20] involves considerable simplifications, modelling the reinforcement layers by the thin beam theory and the matrix as an elastic material using one-dimensional stress analysis.

Fig. 11 gives an example of the critical strain plotted against the fibre volume fraction (logarithmic scale) for the extension mode (the 2nd mode) calculated using the exact solution and 
the model [20]. The plot was computed [11] for the case of composite consisting of two alternating linear-elastic compressible layers. Lines 1, 2, and 3 in the plot correspond to the ratios of Young's modulus of the fibres to the shear modulus of the matrix equal 50, 100 and 200, respectively; the Poisson ratios for both layers were always 0.25 .

It is clear from the plot that the model [20] can give a significant discrepancy in comparison with the exact approach even for the simplest case of linear elastic compressible layers undergoing small pre-critical deformations and considered within the scope of geometrically linear theory. For small fibre volume fractions the approximate approach gives physically unrealistic critical strains. It does not describe the phenomenon under consideration even on the qualitative level, since it predicts a different mode of stability loss from that obtained by the 3-D exact analysis. For more complex models, which take into account large deformations and geometrical and physical non-linearity (e.g. those considered in this chapter), the considered approximate theory is definitely inapplicable and one can expect even a bigger difference between the exact and approximate approaches. The exact approach utilised throughout this paper allows us to take into account large deformations, geometrical and physical non-linearities and load biaxiality that the simplified methods cannot consider.

\subsection{Materials containing elastic-plastic layers}

Now, let us consider the following layered material: the reinforcement behaves as a linearelastic isotropic compressible material, Eq. (29), and the matrix response is elastic-plastic incompressible described by the following relationship for equivalent stress $\left(\sigma_{I}^{0}\right)$ and strain $\left(\varepsilon_{I}^{0}\right)$ :

$$
\sigma_{I}^{0}=A_{m}\left(\varepsilon_{I}^{0}\right)^{k_{m}}
$$

where $k_{m}$ and $A_{m}$ are material constants for elastic-plastic matrix. The constitutive equation (33) is typical for metal matrix composites, see [13, 14, 34, 35]. Again, using the expressions for $\omega_{i j \alpha \beta}$ and $\kappa_{i j \alpha \beta}$ [17], one can deduce the transcendental equations for each of the considered modes of stability loss, see $[13,14]$.

The computed values of critical strain for biaxial and uniaxial loading are presented in Figs. 12-19. Figs. 12-15 correspond to the case of perfectly bonded layers and Figs. 16-19 - to the case of perfectly lubricated layers. The results show how the bonds between the layers affect the solution for the first two modes of stability loss. The 3-D plots illustrate the combined effect of changes in the ratio of layer thicknesses and the material properties of the layers on the critical strains for the $1^{\text {st }}$ and the $2^{\text {nd }}$ modes of stability loss. 
Figs. 12 and 13 give the results for materials with perfectly bonded layers under uniaxial loading. The plots show that an increase in the ratio of coefficient A for the matrix and Young's modulus for the reinforcing layer would lead to a higher critical strain. In the same time, the change in the ratio of layer thicknesses has a non-linear and non-monotonous effect on the critical strain value.

The results for materials with perfectly bonded layers under biaxial loading are shown in Figs. 14 and 15. Similarly to the case of uniaxial compression, the plots illustrate that an increase in the ratio of coefficient A for the matrix and Young's modulus for the reinforcing layer would lead to a higher critical strain. The change in the coefficient $k$ for the matrix has almost no effect on the critical strain for the $2^{\text {nd }}$ mode of stability loss. For the $1^{\text {st }}$ mode of stability loss any increase in the value of coefficient $k$ leads to a decreasing critical strain.

Figs. 16 and 17 correspond to the case of uniaxial compression of materials with perfectly lubricated layers. The plots show the change in the ratio of layer thicknesses has almost no effect on the critical strain for the $2^{\text {nd }}$ mode of stability loss. The critical strain for the $1^{\text {st }}$ mode of stability loss decreases when the ratio of layer thicknesses increases. An increase in the ratio of coefficient A for the matrix and Young's modulus for the reinforcing layer would lead to a higher critical strain. This effect is more pronounced for the $2^{\text {nd }}$ mode of stability loss.

The results for materials with perfectly lubricated layers under biaxial compression are presented in Figs. 18 and 19. Similarly to the case of uniaxial compression, the plots show that an increase in the ratio of coefficient A for the matrix and Young's modulus for the reinforcing layer would lead to a higher critical strain. The change in the coefficient $k$ for the matrix has almost no effect on the critical strain for the $2^{\text {nd }}$ mode of stability loss. For the $1^{\text {st }}$ mode of stability loss any increase in the value of coefficient $k$ leads to a decreasing critical strain.

\subsection{Bounds for the critical controlled parameters}

In this subsection, the critical values of controlled parameters for perfectly bonded and perfectly lubricated layers under different types of loading are compared for hyperelastic materials and materials containing elastic-plastic layers.

According to Eq. (9), these values form the bounds for the critical controlled parameters (i.e. either for critical strains or for critical shortening factors) for materials with imperfections of interfacial adhesion. If for critical strain the bounds have the form of Eq. (9), for critical shortening factors taking into account Eq. (26) they are

$$
\lambda_{c r}^{p b} \leq \lambda_{c r} \leq \lambda_{c r}^{p l} .
$$


In practice, layered materials contain not only interlaminar, but also various sorts of intralaminar defects. The effect of intralaminar damage can be accounted for by considering layers with reduced stiffness properties - see, for example, [36, 37].

The computed results for four modes of stability loss for hyperelastic incompressible layered materials (considered in Subsection 4.2) are shown in Fig. 20 for the case of biaxial loading. The bounds for shortening factor are wider when the ratio of material constants is lower. For the $2^{\text {nd }}$ mode of stability loss the results for perfectly bonded and perfectly lubricated layers are very close when the ratio of material constants reaches a certain value (around 60 for the considered case). For the $1^{\text {st }}$ and the $2^{\text {nd }}$ mode of stability loss, when the ratio of material constants increases, the value of shortening factor increases both for the case of perfectly bonded layers and for perfectly lubricated layers. For the $3^{\text {rd }}$ and the $4^{\text {th }}$ modes of stability loss an increase in the ratio of material constants has an opposite effect of the value of shortening factor: it increases for the case of perfectly bonded layers and decreases for the case of perfectly lubricated layers.

The results of computations for layered materials with elastic-plastic matrix (considered in Subsection 4.5) are shown in Figs. 21 and 22 for the case of biaxial loading. For the $1^{\text {st }}$ and the $2^{\text {nd }}$ modes of stability loss the critical strain remains constant while the ratio of the layer thicknesses is lower than a certain value (around 0.02 for the case of Fig. 21). In this case the problem is effectively reduced to a linear-elastic layer between two elastic-plastic half-spaces, with the critical strain coinciding with the one obtained analytically for the latter case in [13] - yet another verification of the obtained results. For the higher values of the ratio of layer thicknesses it has a strongly non-linear and non-monotonous effect on the critical strain. When the ratio is higher than a certain value (for Fig. 18 this value is around 0.04), the bounds for critical strain for the $1^{\text {st }}$ mode of stability loss become narrower, which is not the case for the $2^{\text {nd }}$ mode of stability loss.

The bounds for critical strain are shown in Fig. 22 as a function of $k_{m}$. With the increase of the coefficient $k_{m}$, the distance between the upper and the lower curves significantly decreases for the $1^{\text {st }}$ mode of stability loss and remains almost the same for the $2^{\text {nd }}$ mode. The change in the coefficient $k_{m}$ has a strongly non-linear and non-monotonous effect on the critical strain for the $2^{\text {nd }}$ mode of stability loss.

The computed bounds appear to give a reasonable estimation for the critical controlled parameters and may be considered as the first approximation on the way to the exact solution of the problem of stability in compression along interfacial defects. Further work is required to compare the results with experimental observations and measurements. 


\section{Discussion}

The purpose of this work was to present a procedure which uniformly deals with different types of layered materials. In the paper the investigation of the internal instability for different types of layered materials, namely hyperelastic incompressible, compressible linear elastic and materials with elastic-plastic layers was conducted. The analysis of different loading schemes and precritical conditions was carried out using the developed software package with fully automated numerical procedure. MATLAB was used to create the software which has graphical user friendly interface and the database of material properties.

We have intentionally chosen to present the computations for model generic material systems only. The applicability of the method to practical materials, e.g., composite materials utilised in aerospace, automotive and other industries, or layers rocks, should be discussed separately of each class of such materials. It would depend on many factors, such as the ability of the equations of Newtonian solid mechanics to fully capture the influence of fine microstructure, various types of defects usually present in real-life materials, the importance of considering more complex loading schemes, etc. In order to take such factors into account, some simplifying assumptions may be required when developing a robust solution. Then the presented analytical solution obtained within the 3-D theory of stability (albeit for a very particular model configuration with a particular loading scheme) can be used as a benchmark for those simplified methods.

The works [38, 39] gave an example of one possible applications of the model presented in this paper. Carbon fibre composite materials are sensitive to open holes, defects and low-velocity impact that can cause barely visible damage (BVID) that can significantly reduce their stiffness and strength properties. To develop structures, which are more damage resistant and tolerant, it is necessary to understand how the damage is caused and how it can affect residual performance. A typical aircraft structure such as a fuselage shell or a wing surface usually consists of a skin reinforced with stiffeners. Most research on open holes and impact damage in carbon-fibre composites are based on testing of small laminates rather than structural elements or full-scale structures. An analytical formula, based on 3-D stability theory, was presented in [38] for calculating the unnotched compressive strength of a multidirectional composite plate. Then the maximum stress failure criterion was employed to estimate the critical load of a stiffened panel with an equivalent open hole loaded in compression. In the range of the model applicability critical loads predicted by the model were very close to the measured data [39]. 


\section{Acknowledgements}

Financial support of the part of this research by The Royal Society, The Royal Academy of Engineering, and The Carnegie Trust for the Universities of Scotland is gratefully acknowledged.

\section{References}

1. Budiansky B, Fleck NA (1994) Compressive kinking of fibre composites: a topical review, Applied Mechanics Reviews, 47( 6), S246-S270.

2. Soutis C, Turkmen D (1995) Influence of shear properties and fibre imperfections on the compressive behaviour of CFRP laminates, Applied Composite Materials, 2(6), 327-342.

3. Schultheisz C, Waas A (1996) Compressive failure of composites, parts I and II, Progress in Aerospace Science, 32(1), 1-78.

4. Niu K, Talreja R (2000) Modelling of compressive failure in fiber reinforced composites, International Journal of Solids \& Structures, 37(17), 2405-2428.

5. Dow NF, Grunfest IJ (1960) Determination of most needed potentially possible improvements in materials for ballistic and space vehicles: General Electric Co., Space Sci. Lab. TISR 60 SD389.

6. Biot MA (1965) Mechanics of incremental deformations. New York, Wiley.

7. Kouri JV, Atluri SN. (1993) Analytical modelling of laminated composites, Composites Science and Technology, 46(4), 335-344.

8. Guz AN (1969) On setting up a stability theory of unidirectional fibrous materials, Soviet Applied Mechanics, 5(2), 156-162.

9. Babich IY, Guz AN (1969) Deformation instability of laminated materials, Soviet Applied Mechanics, 5(3), 53-57.

10. Babich IY, Guz AN (1972) On the theory of elastic stability of compressible and incompressible composite media, Polymer Mechanics, 5.

11. Guz AN, ed. (1992) Micromechanics of composite materials: focus on Ukrainian Research, Applied Mechanics Reviews, 45(2), 15-101.

12. Guz IA (1989) Spatial nonaxisymmetric problems of the theory of stability of laminar highly elastic composite materials, Soviet Applied Mechanics, 25(11), 1080-1085. 
13. Guz IA (1989) Three-dimensional nonaxisymmetric problems of the theory of stability of composite materials with a metallic matrix, Soviet Applied Mechanics, 25(12), 1196-1201.

14. Guz IA (1998) Composites with interlaminar imperfections: substantiation of the bounds for failure parameters in compression, Composites Part B, 29(4), 343-350.

15. Guz IA, Soutis C (2001) A 3-D stability theory applied to layered rocks undergoing finite deformations in biaxial compression, European Journal of Mechanics - A/Solids, 20(1), 139-153.

16. Guz IA, Soutis C (2001) Compressive fracture of non-linear composites undergoing large deformations, International Journal of Solids \& Structures, 38(21), 3759-3770.

17. Guz IA, Herrmann KP (2003) On the lower bounds for critical loads under large deformations in nonlinear hyperelastic composites with imperfect interlaminar adhesion, European Journal of Mechanics, A/Solids, 22(6), 837-849.

18. Menshykova MV, Guz IA, Menshykov OV (2009) A Unified Computational Approach to Instability of Periodic Laminated Materials, Computer Modelling in Engineering \& Sciences (CMES), 51(3), 239259.

19. Guz IA (2005) The effect of the multi-axiality of compressive loading on the accuracy of a continuum model for layered materials. International Journal of Solids and Structures, 42(2), 439-453.

20. Rosen BW (1965) Mechanics of composite strengthening, Fiber Composite Materials, American Society of Metals, Metals Park, ch.3, 37-75.

21. Schuerch H (1966) Prediction of compressive strength in uniaxial boron fibre-metal matrix composite materials, AIAA Journal, 4(1), 102-106.

22. Sadovsky MA, Pu SL, Hussain MA (1967) Buckling of microfibers, Journal of Applied Mechanics, 34(12), 1011-1016.

23. Guz IA, Soutis C (2000) Critical strains in layered composites with interfacial defects loaded in uniaxial or biaxial compression, Plastics, Rubber and Composits, 29(9), 489-495.

24. Soutis C, Guz IA (2001) Predicting fracture of layered composites caused by internal instability, Composites, Part A, 32(9), 1243-1253.

25. Guynn EG, Bradley WL, Ochoa O (1992) A parametric study of variables that affect fibre microbuckling initiation in composite laminates: part 1- analyses, part 2-experiments, Journal of Composite Materials, 26(11), 1594-1627.

26. Moran P, Liu L, Shih C (1995) Kinking band formation and band broadening in fibre composites under compressive loading, Acta Metallutgica at Materialia, 43(8), 2943-2958.

27. Berbinau P, Soutis C, Guz IA (1999) Compressive failure of $0^{\circ}$ unidirectional carbon-fibre-reinforced plastic (CFRP) laminates by fibre microbuckling. Composites Science and Technology, 59(9), 14511455.

28. Winiarski B, Guz IA (2008) The effect of fibre volume fraction on the onset of fracture in laminar materials with an array of coplanar interface cracks, Composites Science and Technology, 68(12), 2367-2375. 
29. Guz AN (1999) Fundamentals of the three-dimensional theory of stability of deformable bodies, BerlinHeidelberg, Springer-Verlag.

30. Aboudi J (1987) Damage in composites - modelling of imperfect bonding, Composite Science and Technology, 28(2), 103-128.

31. Librescu L, Schmidt R (2001) A general linear theory of laminated composite shells featuring interlaminar bonding imperfections, International Journal of Solids \& Structures, 38(19), 3355-3375.

32. Soutis C, Guz IA (2006) Fracture of layered composites by internal fibre instability: Effect of interfacial adhesion. The Aeronautical Journal, 110(1105), 185-195.

33. Ling XW, Atluri SN (2007) A hyperelastic description of single wall carbon nanotubes at moderate strains and temperatures, CMES: Computer Modeling in Engineering \& Sciences, 21(1), 81-91.

34. Honeycombe RWK (1968) The plastic deformation of metals, London, Edward Arnold.

35. Pinnel MR, Lawley A (1970) Correlation of yielding and structure in aluminium-stainless steel composites, Metallurgical Transactions, 1(5), 1337-1348.

36. Kashtalyan M, Soutis C (2001) Strain energy release rate for off-axis ply cracking in laminated composites, International Journal of Fracture, 112(2), L3-L8.

37. Kashtalyan M, Soutis C (2006) Modelling off-axis ply matrix cracking in continuous fibre-reinforced polymer matrix composite laminates. Journal of Materials Science, 41(20), 6789-6799.

38. Zhuk Y, Soutis C, Guz IA (2001) Behaviour of thin-skin stiffened CFRP panels with a stress concentrator under in-plane compression. Composites Part B, 32(8), 697-709.

39. Zhuk Y, Soutis C, Guz IA (2002) Stiffened composite panels with a stress concentrator under in-plane compression. International Applied Mechanics, 38(2), 240-252. 


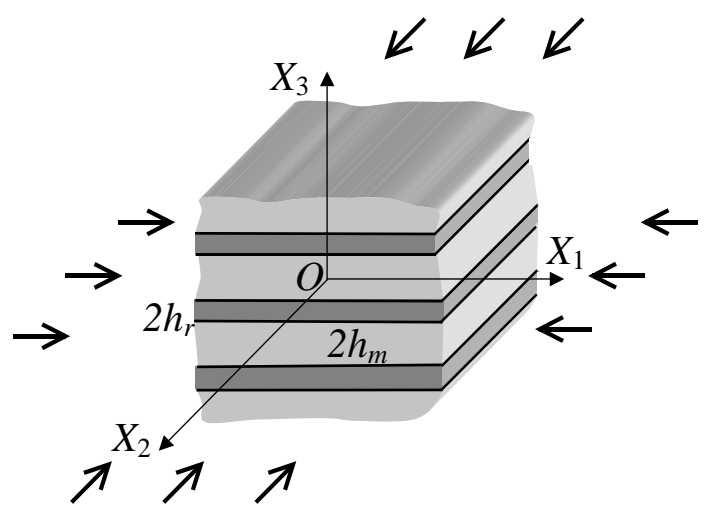

Figure 1. Biaxial compression of a layered material

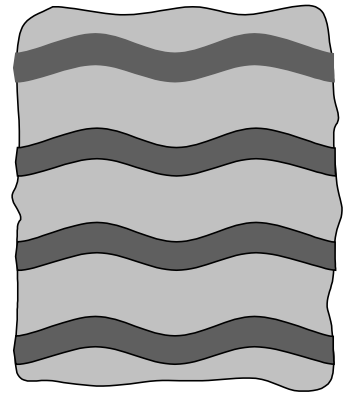

(a)

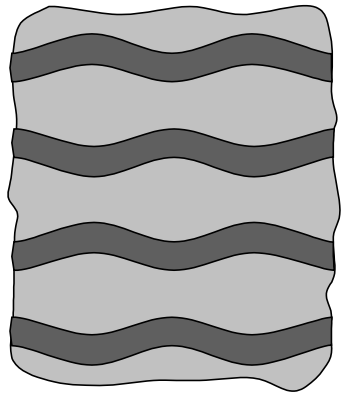

(b)

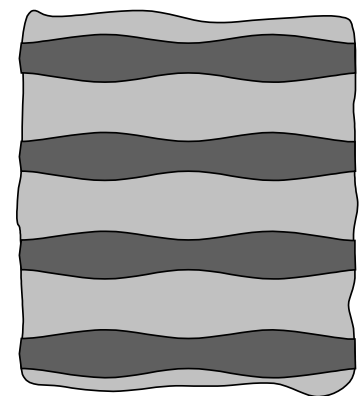

(c)

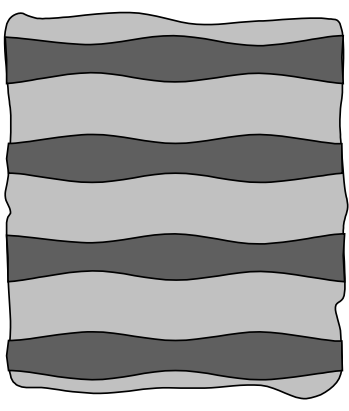

(d)

Figure 2. Modes of stability loss (microbuckling): (a) the 1st (shear) mode; (b) the 2nd (extension) mode, (c) the 3rd mode, (d) the 4th mode 


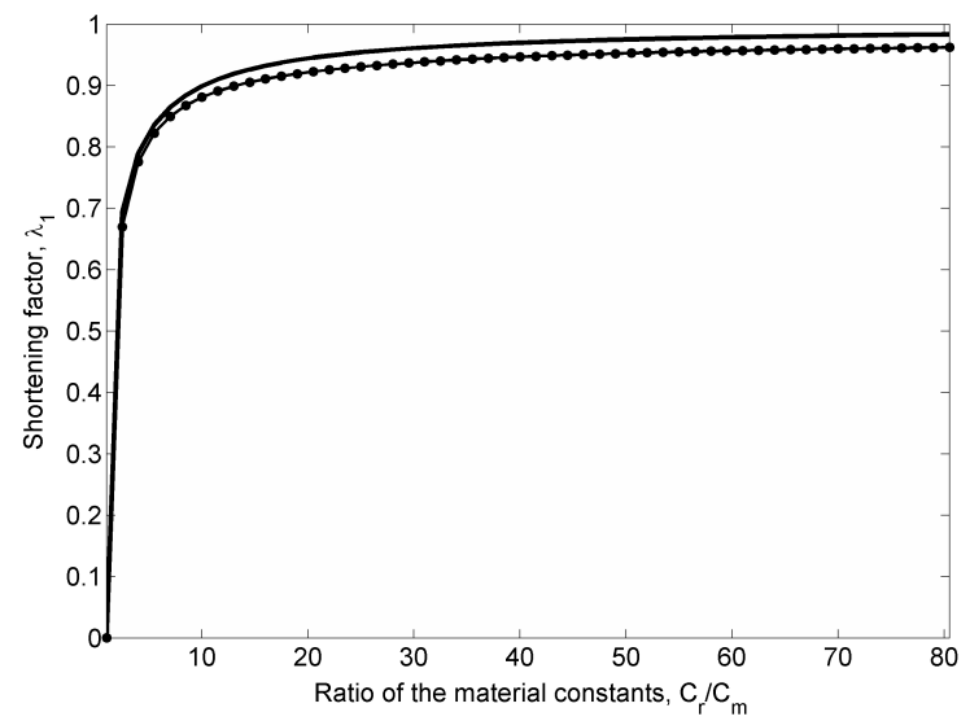

Figure 3. Shortening factor plotted against the ratio of material constants; $h_{r} / h_{m}=0.2$.

Solid line - the $1^{\text {st }}$ (shear) mode of stability loss; marked line - the $2^{\text {nd }}$ (extensional) mode of stability loss.

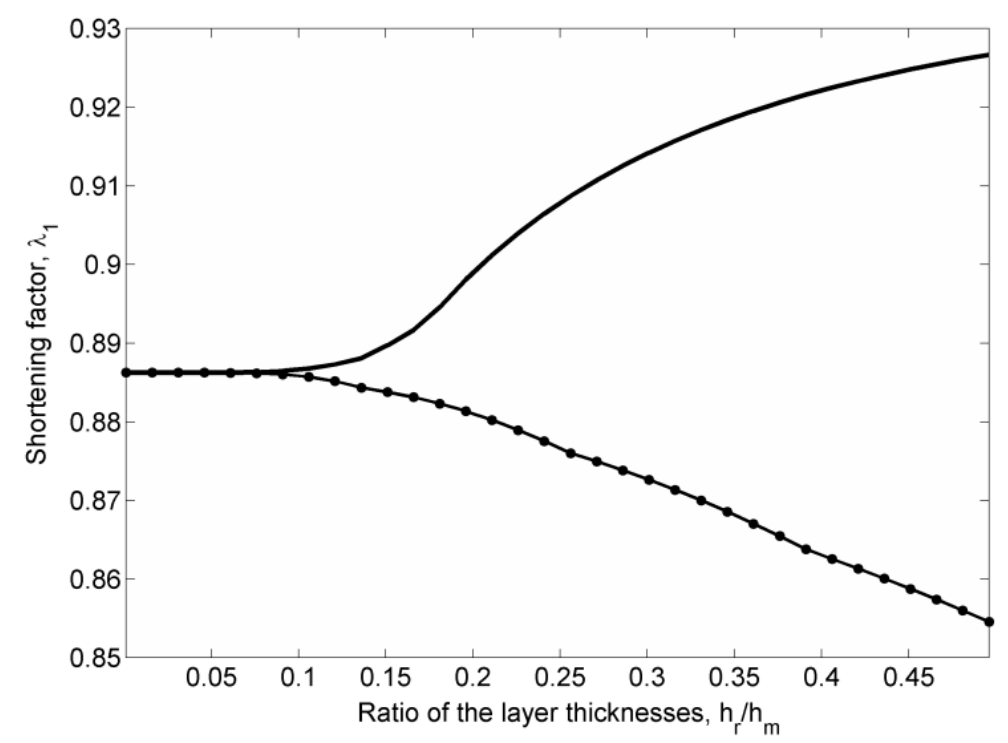

Figure 4. Shortening factor plotted against the ratio of the layer thicknesses; $C_{r} / C_{m}=10$.

Solid line - the $1^{\text {st }}$ (shear) mode of stability loss; marked line - the $2^{\text {nd }}$ (extensional) mode of stability loss. 


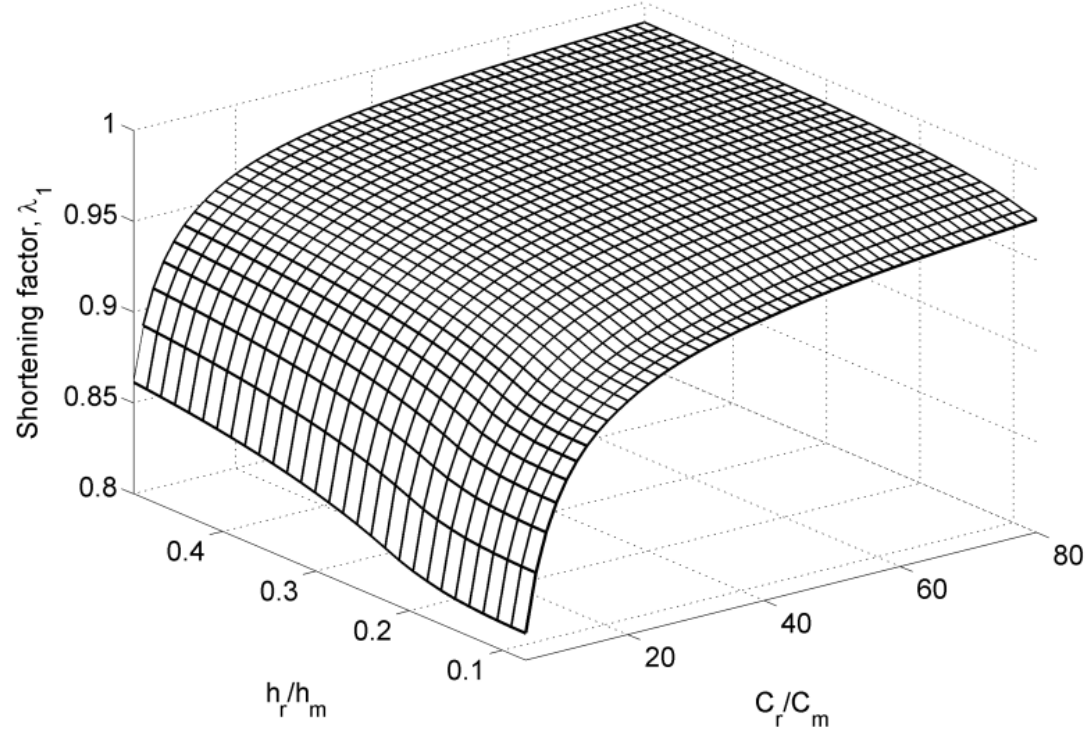

Figure 5. Hyperelastic incompressible layered material with perfectly bonded layers under biaxial loading. The $1^{\text {st }}$ mode of stability loss.

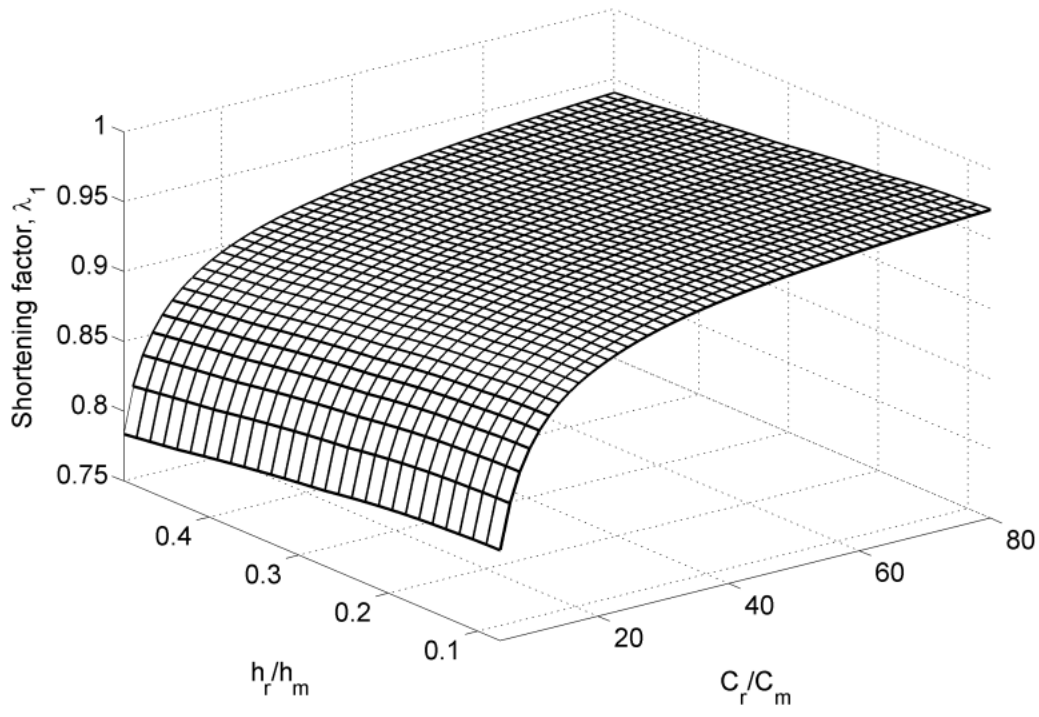

Figure 6. Hyperelastic incompressible layered material with perfectly bonded layers under biaxial loading. The $2^{\text {nd }}$ mode of stability loss. 


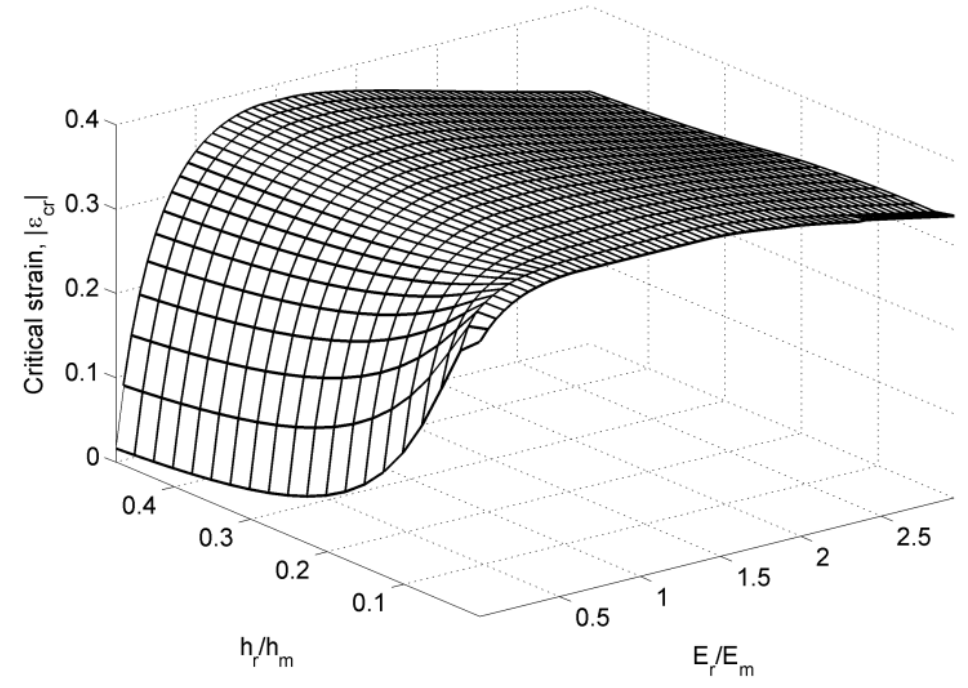

Figure 7. Compressible linear elastic layered material with perfectly bonded layers under uniaxial loading. The $1^{\text {st }}$ mode of stability loss $\left(v_{r}=0.24, v_{m}=0.237\right)$.

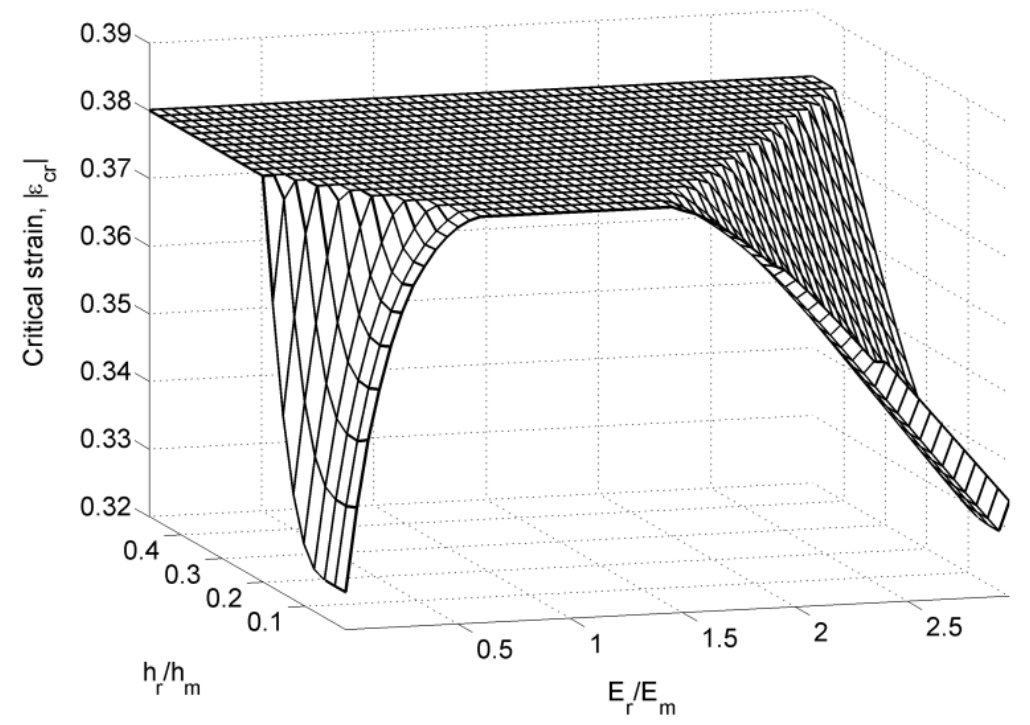

Figure 8. Compressible linear elastic layered material with perfectly bonded layers under uniaxial loading. The $2^{\text {nd }}$ mode of stability loss $\left(v_{r}=0.24, v_{m}=0.237\right)$. 


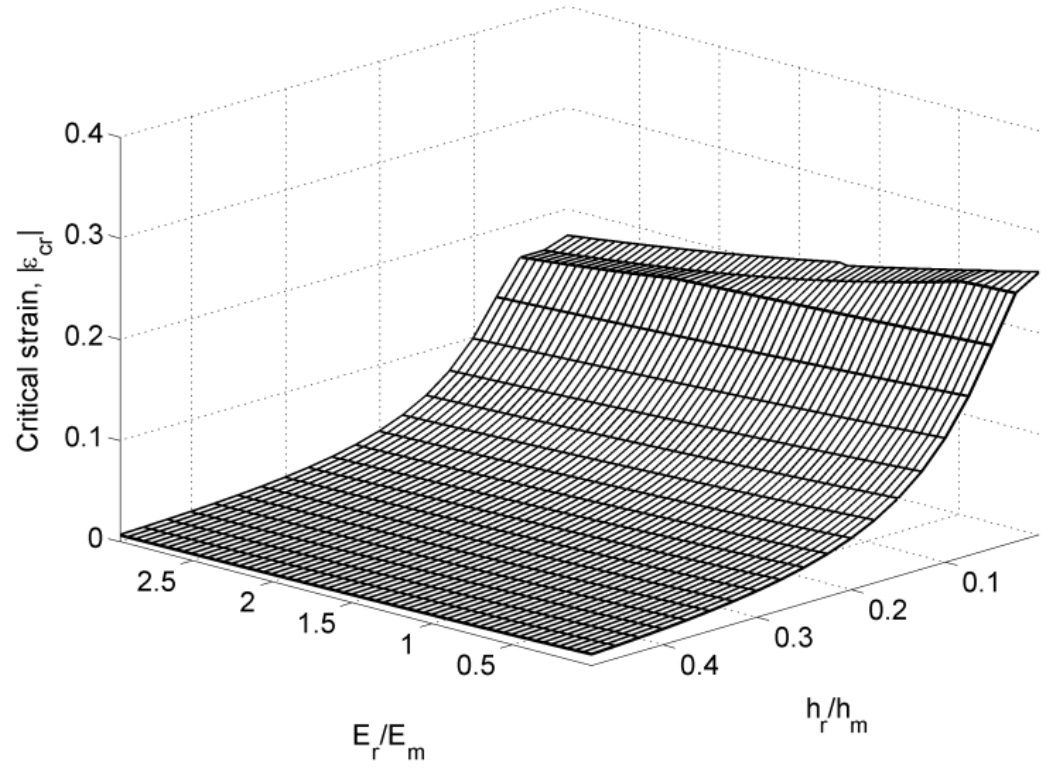

Figure 9. Compressible linear elastic layered material with perfectly lubricated layers under biaxial loading. The $1^{\text {st }}$ mode of stability loss $\left(v_{r}=0.24, v_{m}=0.237\right)$.

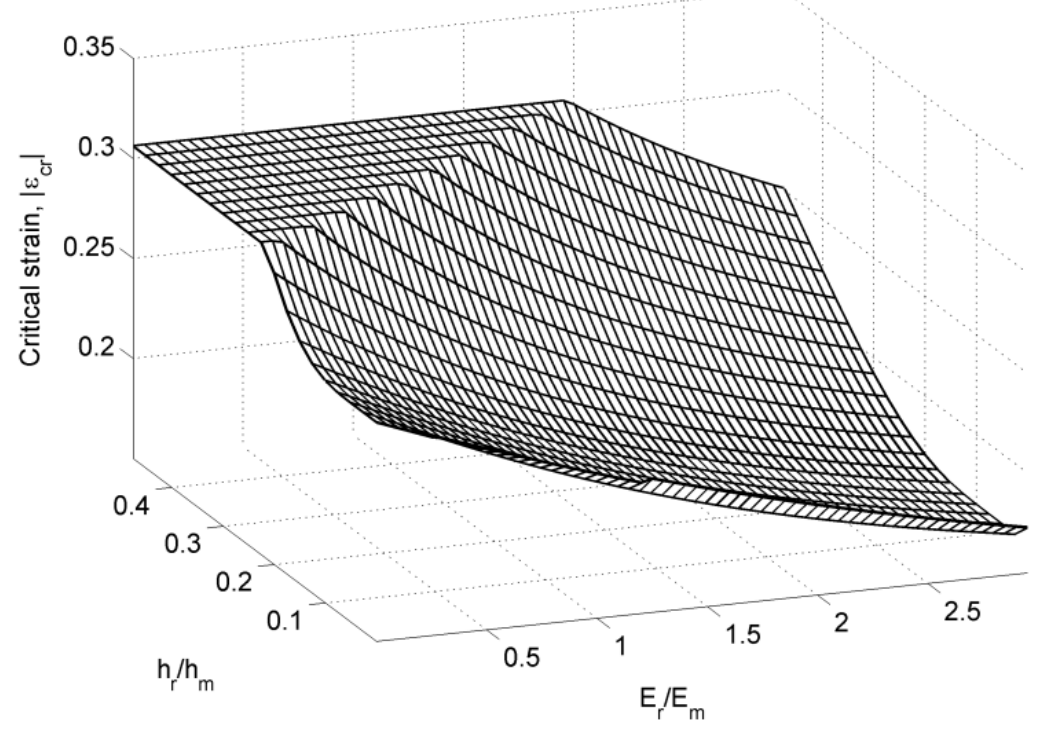

Figure 10. Compressible linear elastic layered material with perfectly lubricated layers under biaxial loading. The $2^{\text {nd }}$ mode of stability loss $\left(v_{r}=0.24, v_{m}=0.237\right)$. 


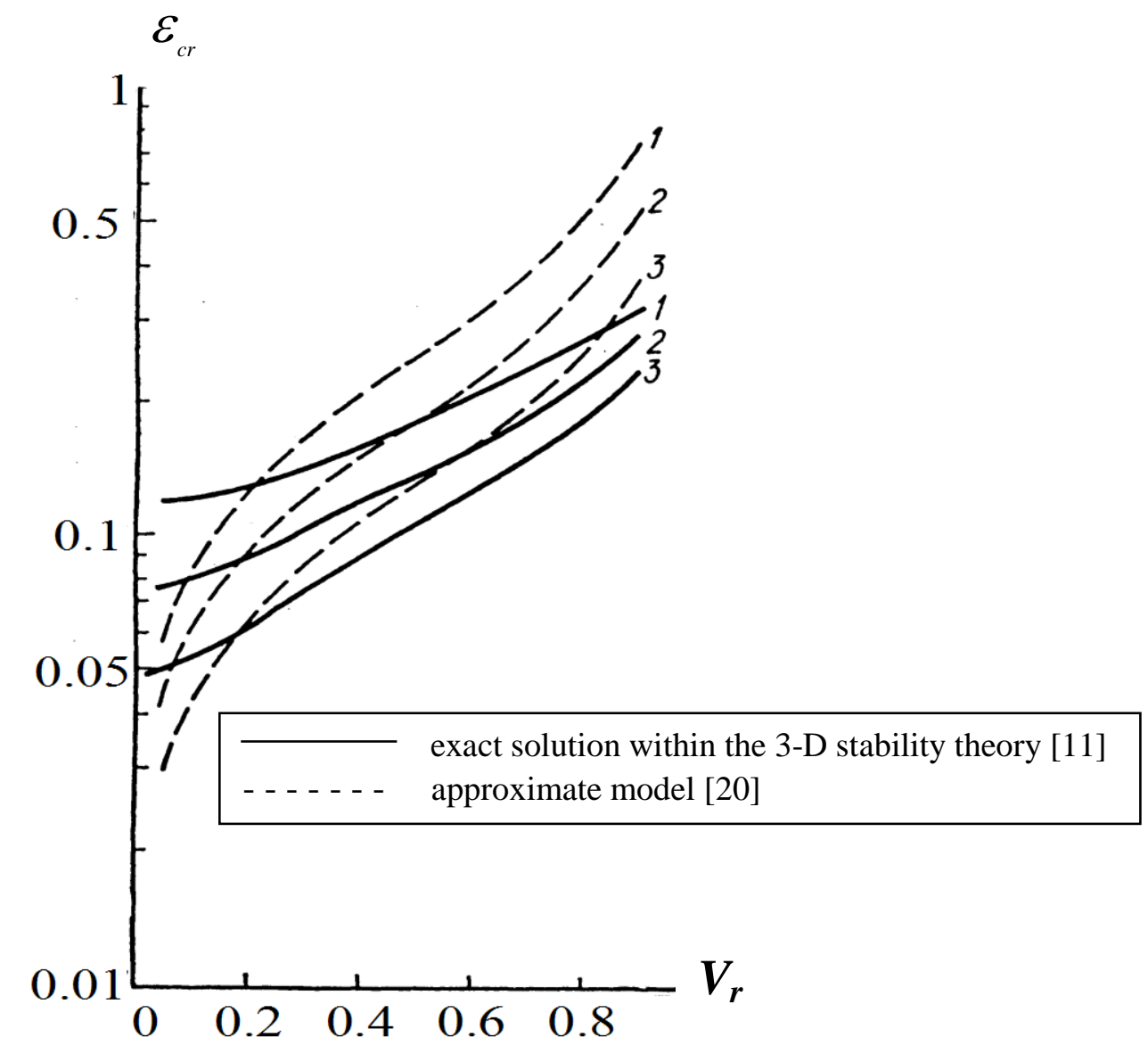

Figure 11. Critical strain plotted against fibre volume fraction for the extension mode (the $2^{\text {nd }}$ mode of stability loss); logarithmic scale. 


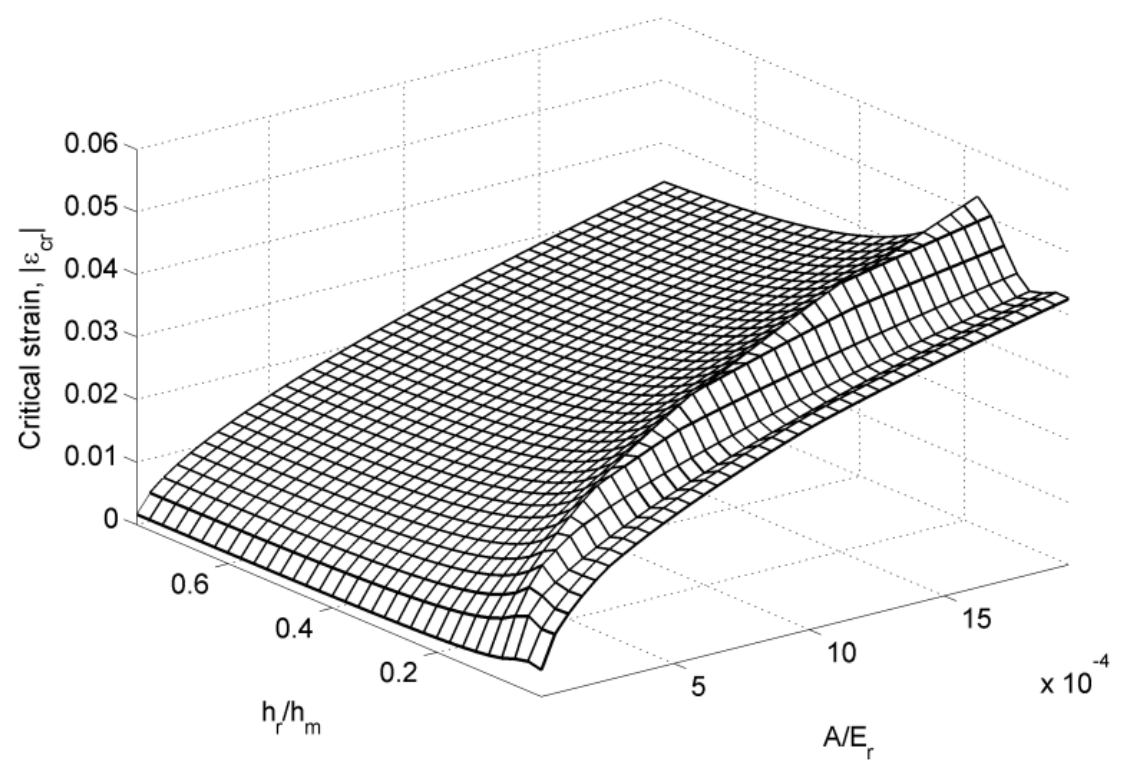

Figure 12. Material containing elastic-plastic perfectly bonded layers under uniaxial loading. The $1^{\text {st }}$ mode of stability loss $\left(k_{m}=0.23, v_{r}=0.2\right)$.

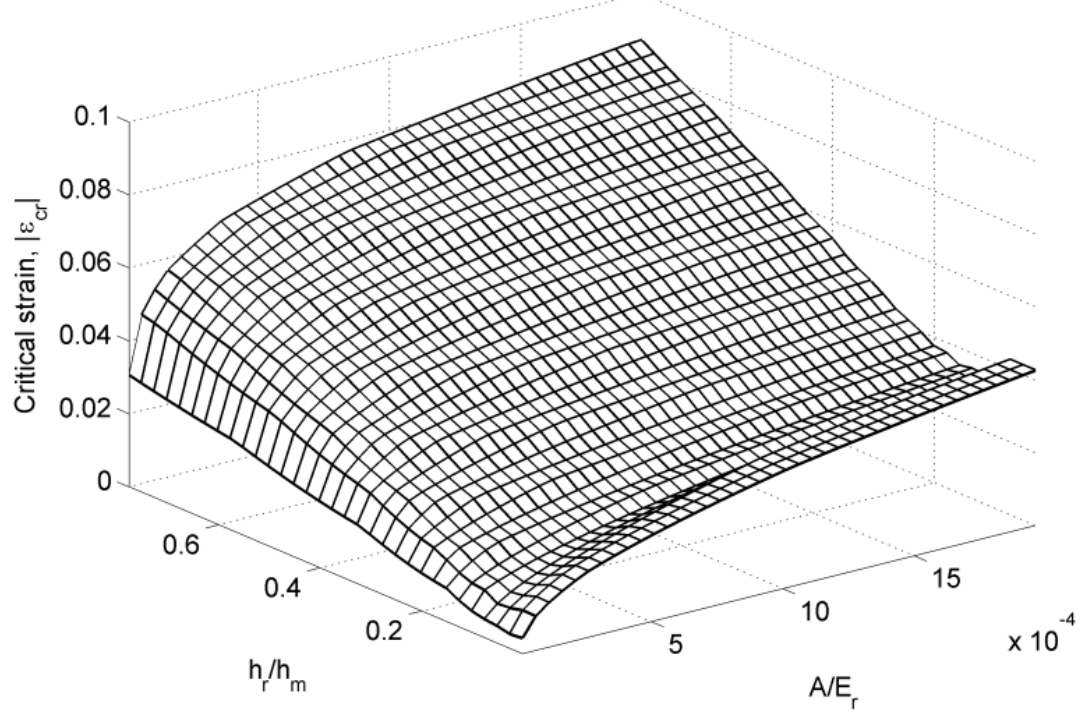

Figure 13. Material containing elastic-plastic perfectly bonded layers under uniaxial loading. The $2^{\text {nd }}$ mode of stability loss $\left(k_{m}=0.23, v_{r}=0.2\right)$. 


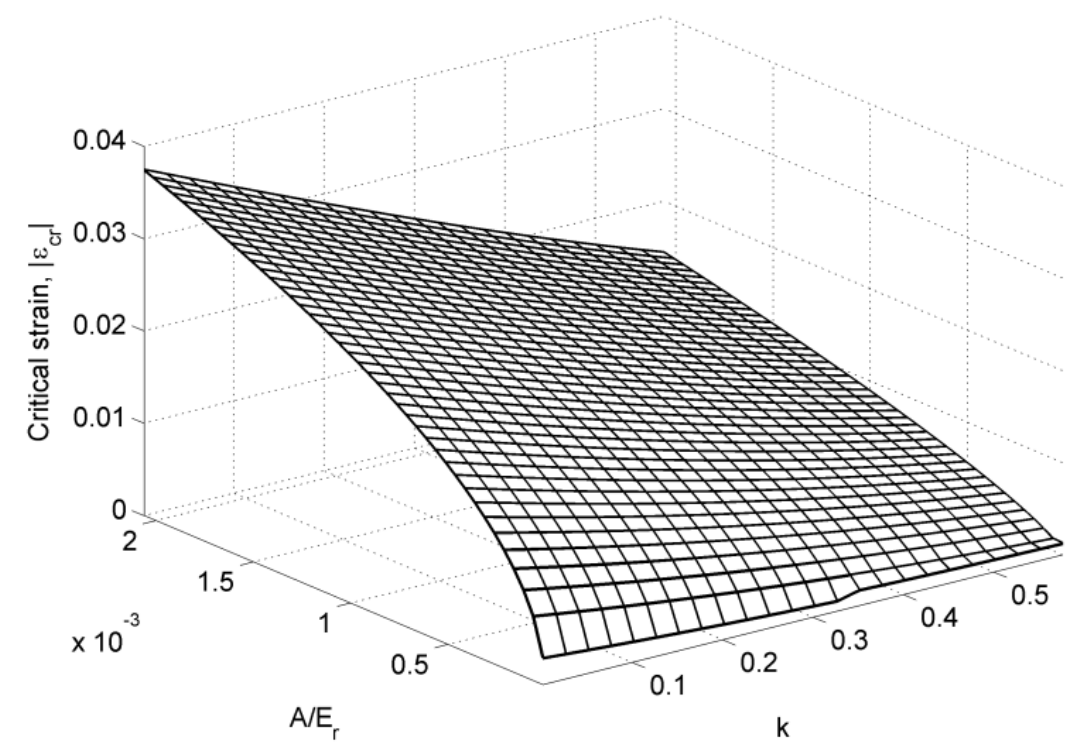

Figure 14. Material containing elastic-plastic perfectly bonded layers under biaxial loading. The $1^{\text {st }}$ mode of stability loss $\left(v_{r}=0.2, h_{r} / h_{m}=0.25\right)$.

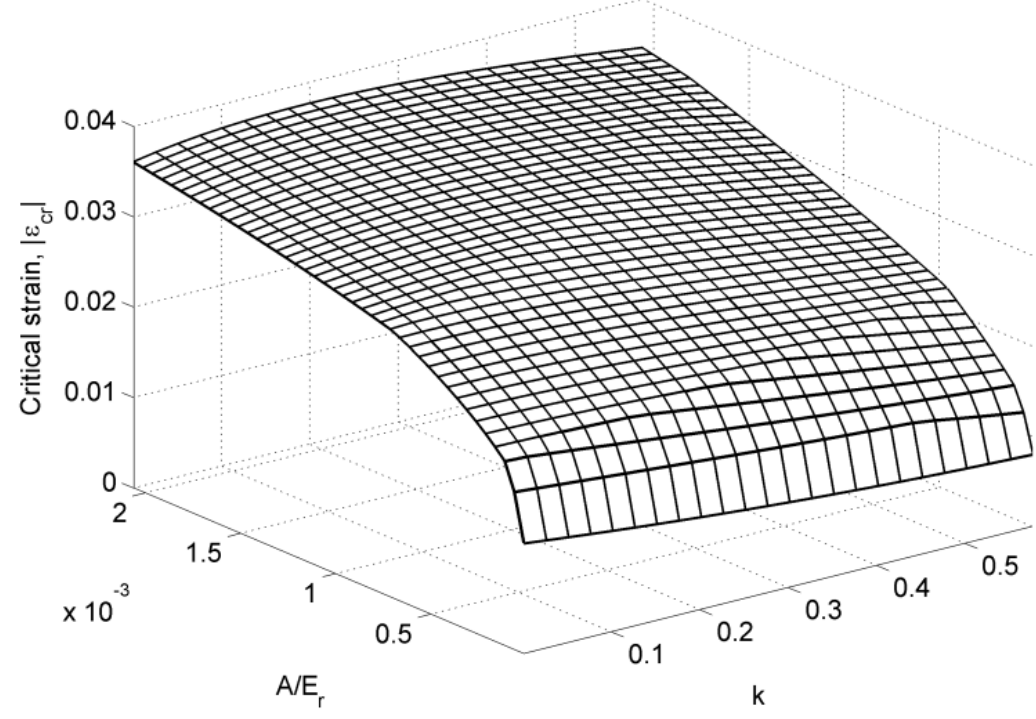

Figure 15. Material containing elastic-plastic perfectly bonded layers under biaxial loading. The $2^{\text {nd }}$ mode of stability loss $\left(v_{r}=0.2, h_{r} / h_{m}=0.25\right)$. 


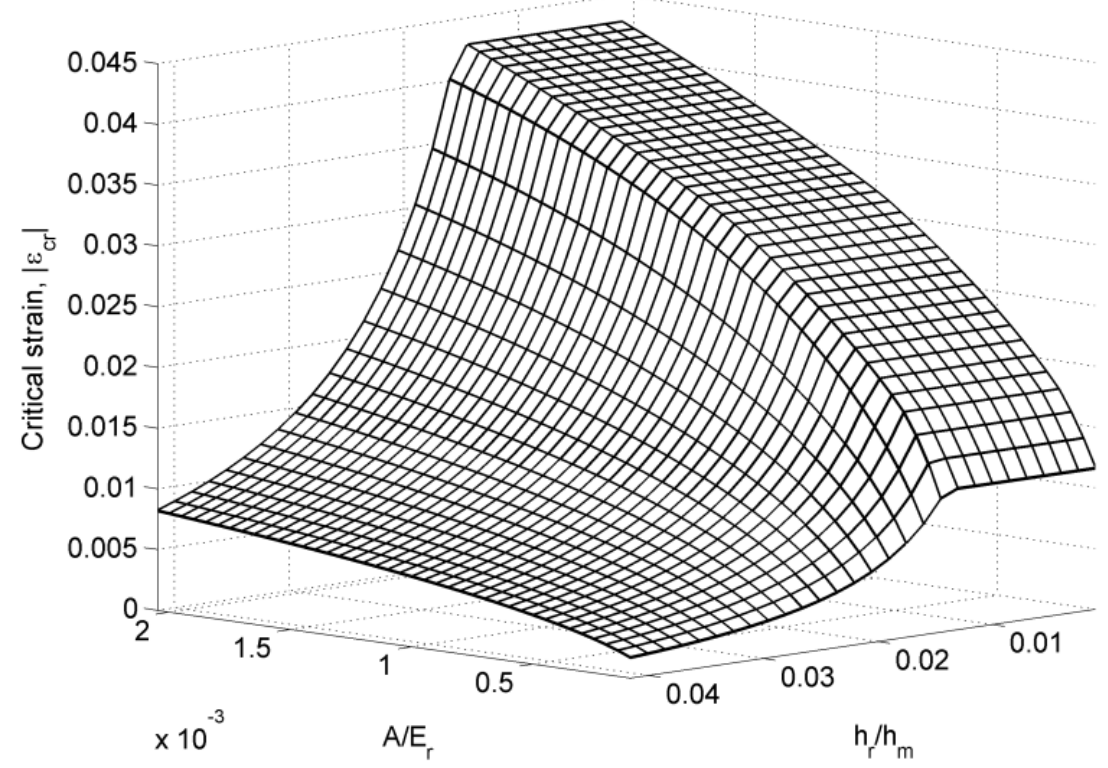

Figure 16. Material containing elastic-plastic perfectly lubricated layers under uniaxial loading.

The $1^{\text {st }}$ mode of stability loss $\left(k_{m}=0.23, v_{r}=0.2\right)$.

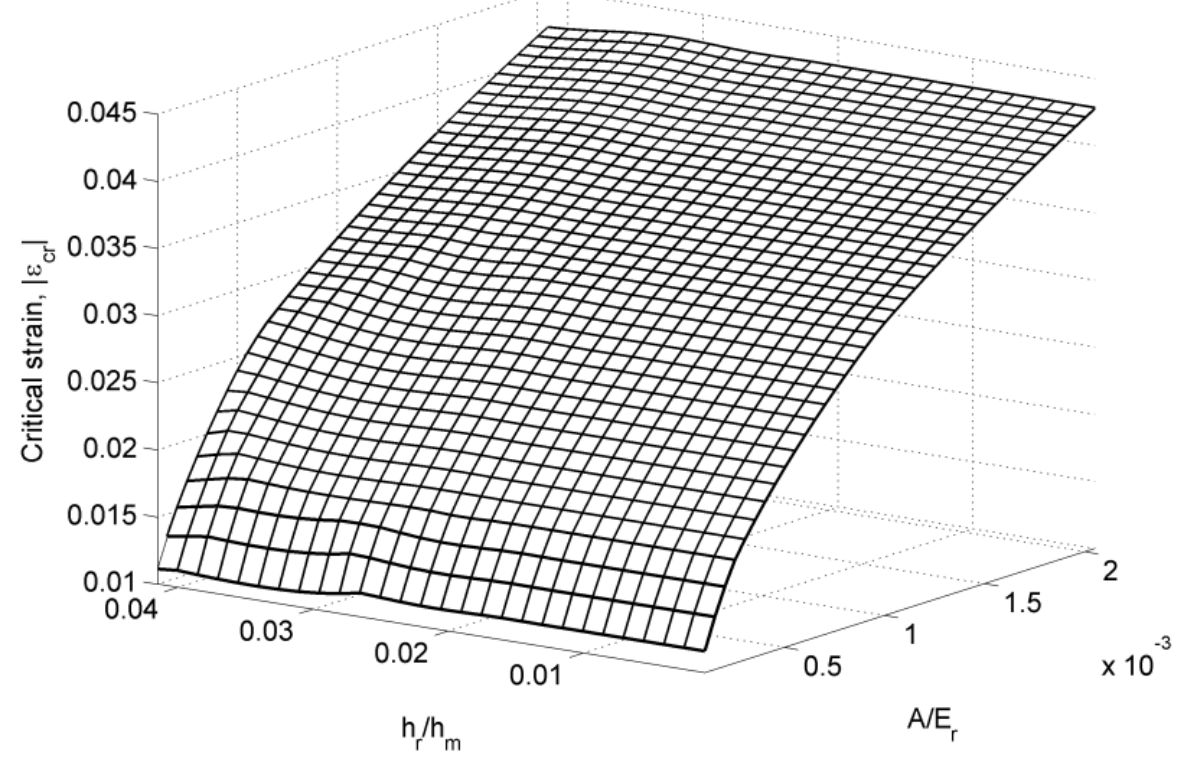

Figure 17. Material containing elastic-plastic perfectly lubricated layers under uniaxial loading. The $2^{\text {nd }}$ mode of stability loss $\left(k_{m}=0.23, v_{r}=0.2\right)$. 


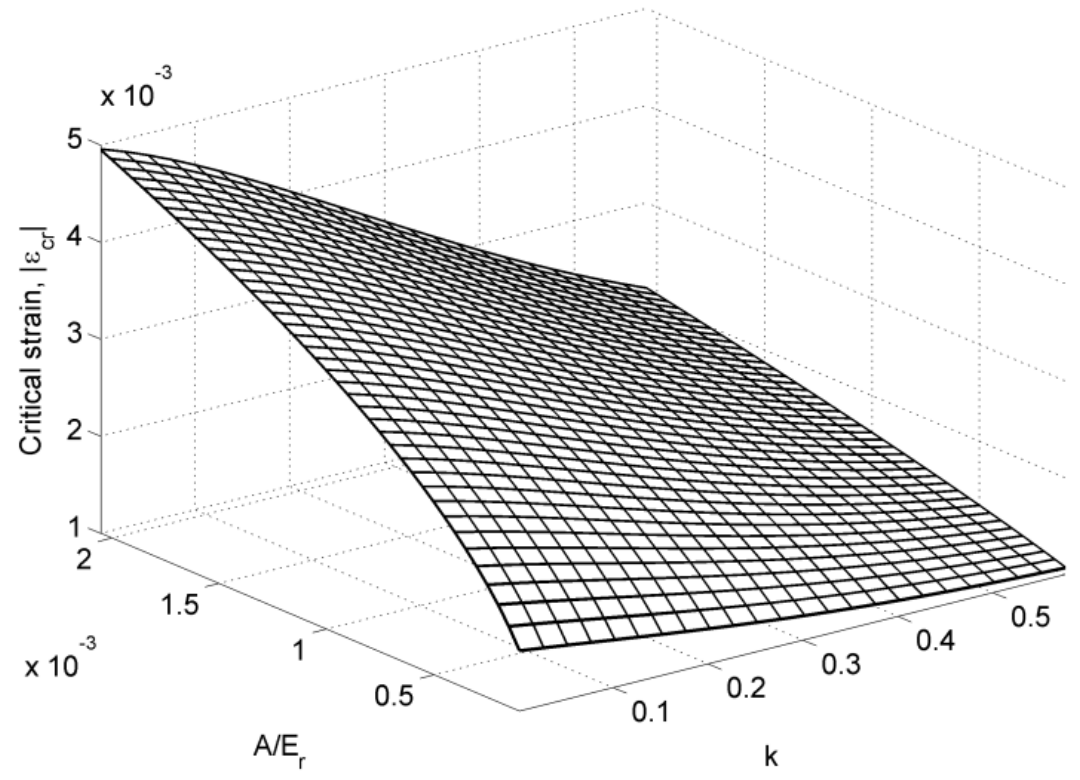

Figure 18. Material containing elastic-plastic perfectly lubricated layers under biaxial loading. The $1^{\text {st }}$ mode of stability loss $\left(v_{r}=0.2, h_{r} / h_{m}=0.25\right)$.

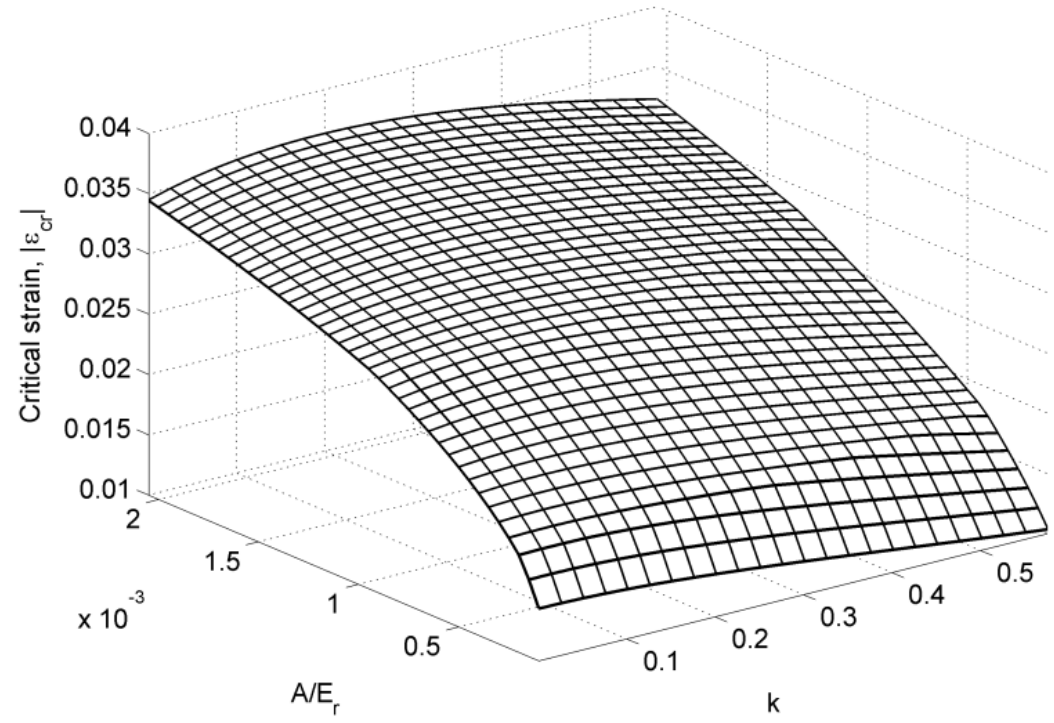

Figure 19. Material containing elastic-plastic perfectly lubricated layers under biaxial loading. The $2^{\text {nd }}$ mode of stability loss $\left(v_{r}=0.2, h_{r} / h_{m}=0.25\right)$. 


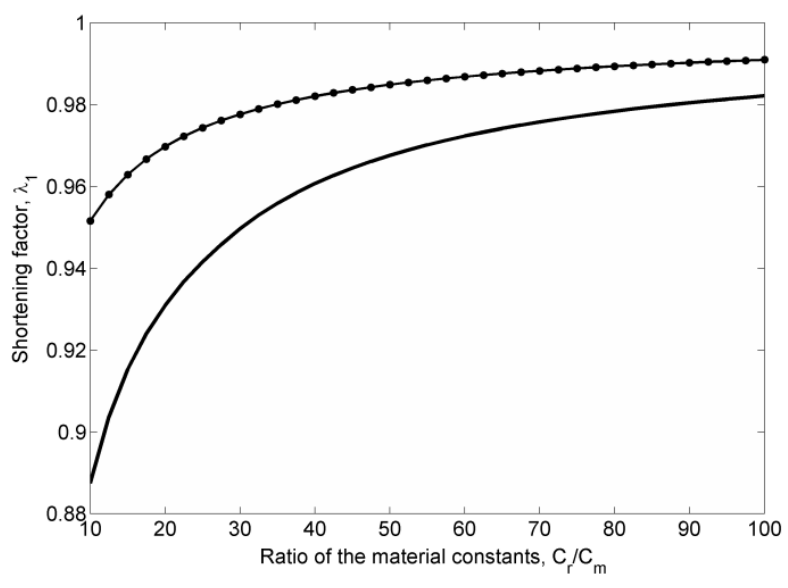

1st mode of stability loss

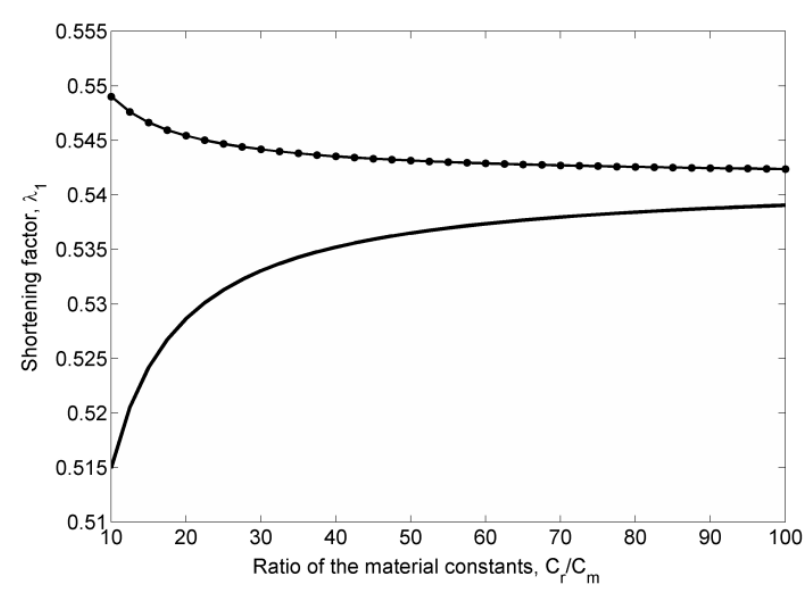

3rd mode of stability loss

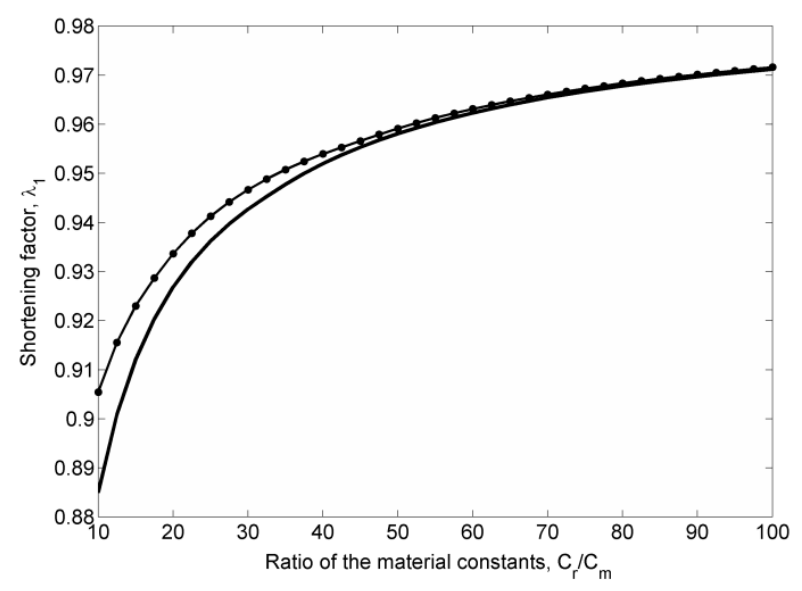

2nd mode of stability loss

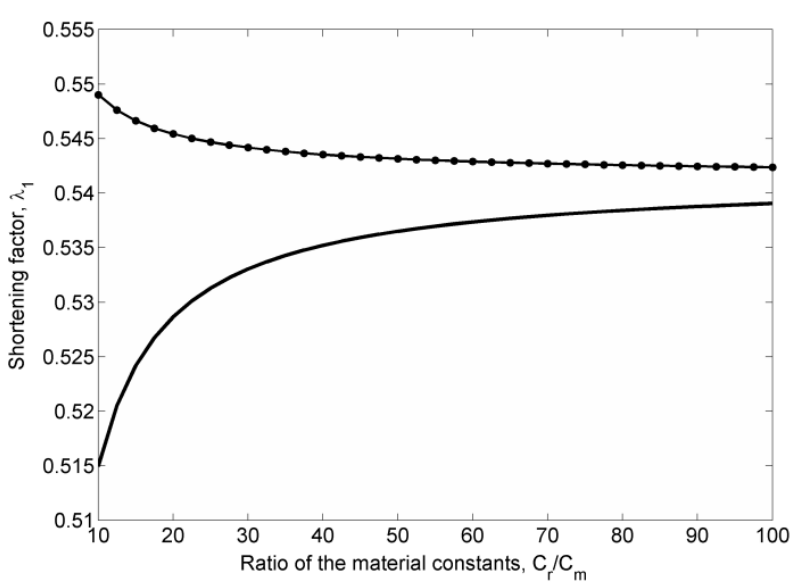

4th mode of stability loss

Figure 20. The bounds for different modes of instability for hyperelastic layered materials under biaxial loading: $h_{r} / h_{m}=0.125$. Solid line - perfectly lubricated layers; marked line - perfectly bonded layers. 

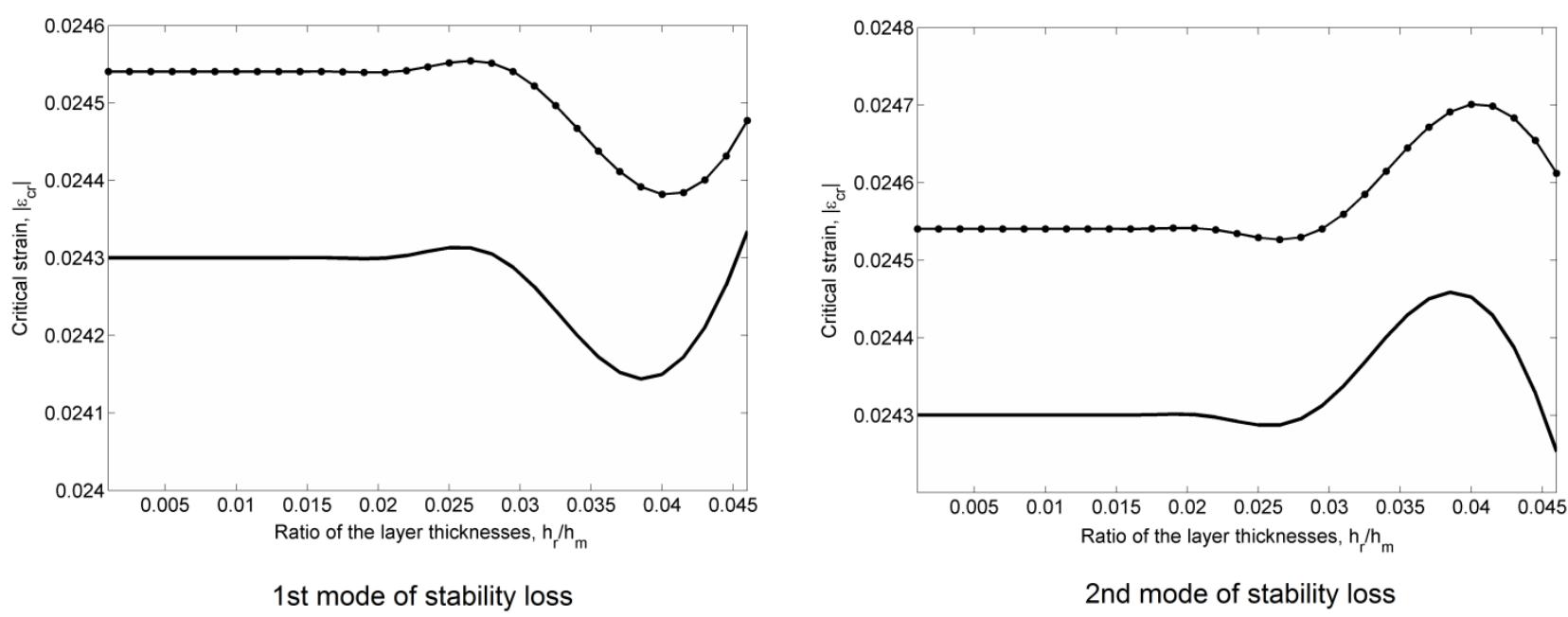

Figure 21. The bounds for the $1^{\text {st }}$ and the $2^{\text {nd }}$ modes of instability for materials containing elasticplastic layers under biaxial loading; $A_{m} / E=0.001, v_{r}=0.237, k_{m}=0.23$.

Solid line - perfectly lubricated layers; marked line- perfectly bonded layers.

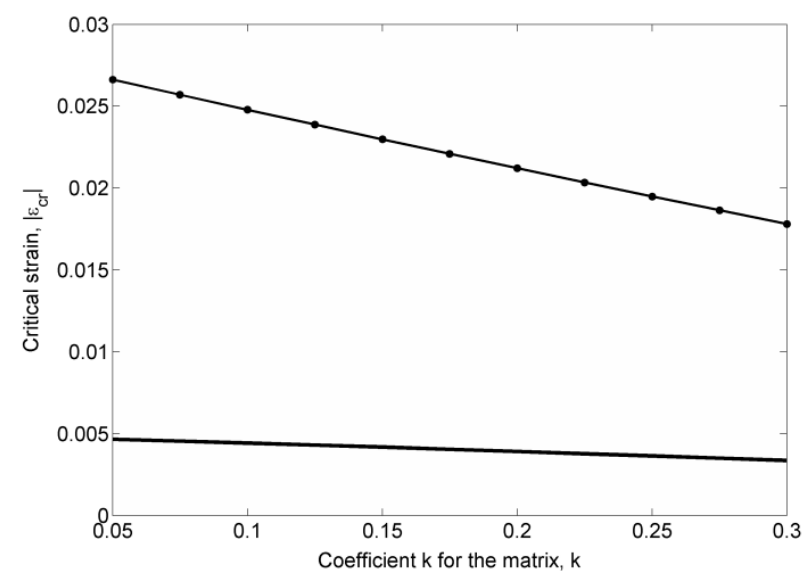

1st mode of stability loss

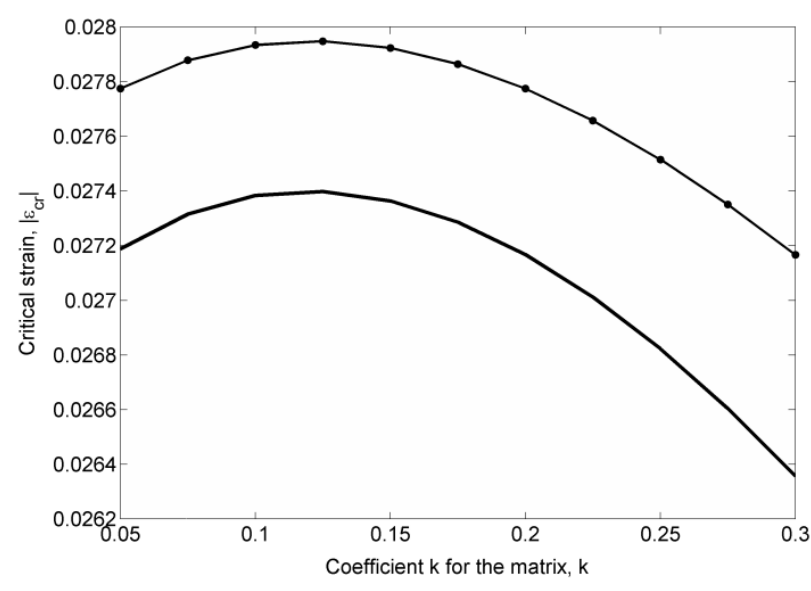

2nd mode of stability loss

Figure 22. The bounds for the $1^{\text {st }}$ and the $2^{\text {nd }}$ modes of instability for materials containing elasticplastic layers under biaxial loading; $A_{m} / E=0.00075, v_{r}=0.23, h_{r} / h_{m}=0.02$.

Solid line - perfectly lubricated layers; marked line - perfectly bonded layers. 